\title{
A hybrid gradient-based/metaheuristic method for Eurocode-compliant size, shape and topology optimization of steel structures
}

\author{
Wouter Dillen ${ }^{\mathrm{a}, *}$, Geert Lombaert ${ }^{\mathrm{b}}$, Mattias Schevenels ${ }^{\mathrm{a}}$ \\ ${ }^{a}$ KU Leuven, Faculty of Engineering Science, Department of Architecture, Kasteelpark \\ Arenberg 1, 3001 Leuven, Belgium \\ ${ }^{b}$ KU Leuven, Faculty of Engineering Science, Department of Civil Engineering, Kasteelpark \\ Arenberg 40, 3001 Leuven, Belgium
}

\begin{abstract}
The production and processing of building materials is responsible for a significant share of global greenhouse gas emissions. Nevertheless, in current building practice, a lot of material is wasted, because load-bearing structures are often grossly overdimensioned. Numerical optimization tools have the potential to reduce the consumption of structural materials, but the lack of customized algorithms prohibits their use in daily design practice. This is because real-life structural optimization problems are complex, involving both discrete and continuous design variables as well as large numbers of building code constraints. In the literature, such problems are usually solved using metaheuristic (often genetic) algorithms, although gradient-based algorithms are better suited for continuous design variables. In this article, a new hybrid gradient-based/metaheuristic algorithm is proposed. It combines gradient-based and metaheuristic methods in a nested approach, exploiting the strengths of both: the ability to handle discrete design variables and fast convergence in terms of continuous design variables. The applicability and usefulness of the new hybrid algorithm is demonstrated in two realistic case studies to minimize the weight of the structure, taking into account all relevant design rules of Eurocode 3. Within identical computing time, the hybrid algorithm obtains significant material savings compared to a conventional genetic algorithm.
\end{abstract}

Keywords: Structural optimization, Eurocode design, Real-life optimization, Genetic algorithm, Sequential Quadratic Programming, Active set

\footnotetext{
* Corresponding author

Email address: wout.dillen@kuleuven.be (Wouter Dillen)
} 


\section{Introduction}

The built environment has an enormous impact on the natural environment. In the EU, it is responsible for $50 \%$ of the consumption of natural resources, $42 \%$ of the total energy consumption, $35 \%$ of greenhouse gas emissions, and $32 \%$ of waste flows [1]. In the recent past, much attention has been paid to reducing the operational environmental impact of buildings. However, studies have shown that half of the greenhouse gas emissions from the built environment are attributed to the embodied impact of buildings, related to the production, transport, construction and disposal of building materials [2, 3], a further half of which is related to structural building materials [4].

In modern building practice, under-utilization of structural materials for rationalization purposes is common [5]. Structural designers often trade in structural efficiency for spare capacity in order to reduce engineering efforts as well as fabrication and construction costs, e.g. by copying sections from the most loaded beam to the other beams, or by using the same sections for different members to avoid confusion on the construction site. While a spare capacity of $5 \%$ to $20 \%$ is considered acceptable among structural engineers $[6,7]$, a recent study by Moynihan and Allwood [5] shows that the spare capacity is as high as $50 \%$ in reality, leaving room for substantial material savings.

Numerical optimization tools have the potential to restore the balance between material efficiency and design rationalization while simultaneously automating tedious parts of the design process (iterative manual selection of member profiles). However, despite the vast body of academic knowledge developed in the past half century, numerical optimization does not easily find its way into the construction sector. According to Rolvink et al. [8], this is due to the fact that the available optimization techniques cannot cope with all characteristics of real-life design problems, do not converge within a reasonable time frame, and/or are not user-friendly. A possible cause is the fact that the academic literature on structural optimization is dominated by a small number of frequently recurring test problems that are not representative of a realistic design situation [9]. The development of an effective optimization method for real-life structural engineering problems remains an important scientific challenge.

Structural optimization methods aim to minimize a certain objective value (e.g. material use) while satisfying a set of constraints (e.g. limitation of stresses, displacements, risk of buckling). Generally, three optimization strategies are distinguished: (1) size optimization, where the aim is to find the optimal dimensions of structural components (e.g. beam sections or plate thicknesses), (2) shape optimization, where the shape of the structure is modified for optimal performance, and (3) topology optimization, where the optimal spatial distribution of structural material or structural components (e.g. bars or braces) is determined. Shape optimization typically involves continuous design variables, as the shape variables can assume any real value within a given range. Size and topology optimization typically involves discrete design variables, which represent

the index of a section in a commercial section catalog, or the presence/absence of a member at a particular location in the structure. The combination of size, 
shape and topology optimization implies a mixture of discrete and continuous design variables, which makes the optimization problem harder to solve.

Optimization methods can be divided into different categories, including gradient-based, metaheuristic and hybrid methods. Depending on the type of design variables, certain optimization methods will be more effective than others.

Gradient-based optimization methods, such as the Sequential Quadratic Programming method [10], the Interior-Point method [11] and the Method of Moving Asymptotes [12], have long been used to solve continuous optimization problems [13]. These algorithms use the sensitivities (gradients) of the objective and constraint functions to determine the search direction towards optimal performance, making them highly efficient. However, because gradient-based methods require the objective and constraint functions to be differentiable, they can only cope with continuous design variables (e.g. shape variables). For discrete problems, the design variables must be relaxed and a penalty scheme or rounding strategy must be used in order to obtain discrete results. While penalization schemes are the preferred choice for continuum topology optimization problems [14], they are not effective for discrete size optimization problems with large section catalogs.

Since 1990, the literature on discrete size optimization is dominated by metaheuristic optimization methods [9], such as Genetic Algorithms [15], Particle Swarm Optimization [16], Simulated Annealing [17], Adaptive Dimensional Search [18] and Monitored Convergence Curve [19]. Metaheuristic methods are typically stochastic in nature, improving a randomly generated set of initial designs in a pseudo-random fashion. They are applicable to virtually all types of optimization problems, including discrete size optimization problems with complex constraints and large section catalogs. Furthermore, they are non-intrusive, in the sense that they can be combined with existing structural analysis software without changing the code, as long as the software can be called via the command line or via an Application Programming Interface (API). However, metaheuristic methods also have important drawbacks: they suffer from the curse of dimensionality [20], they fail to converge well for high-dimensional problems, and they usually involve algorithmic control parameters that are difficult to tune. In addition, metaheuristic methods are often studied without statistical convergence assessment, which makes it difficult to assess the optimality of the result or the effectiveness of the method [9].

Several hybrid optimization methods have been proposed in the literature, e.g. by Einabadi and Ebadzadeh [21], Plevris and Papadrakakis [22], Chen et al. [23], Das et al. [24] and Noel and Jannett [25]. They usually consist of a metaheuristic method that determines a loose estimate of the optimum, which is then further refined by a gradient-based algorithm. However, all existing hybrid methods are intended for either discrete or continuous design variables and are unable to cope with both.

The literature contains a limited number of articles on Eurocode-based optimization at building level. Gutkowski et al. [26] have proposed a heuristic method that recursively analyzes a structure while removing redundant ma- 
terial, which is applied to the 10-bar truss and a simple frame structure in Guerlement et al. [27], considering strength and displacement constraints from Eurocode 3. Jalkanen [28] applied several metaheuristic methods (simulated annealing, tabu search, genetic algorithms and particle swarm optimization) to size, shape and topology optimization of tubular trusses subject to the constraints in Eurocode 3. Mela [29] integrates member constraints specified by Eurocode 3 in the mixed-integer linear programming (MILP) formulation for truss topology design. Van Mellaert et al. extend the MILP approach to joint constraints (limited to size optimization) [30] and apply the approach to global discrete sizing optimization of frame structures [31]. Finally, D'Amico and Pomponi [32] present a fully utilized design-like heuristic method for minimizing material consumption in steel frames with a rectangular layout and constant span lengths.

This article presents a new hybrid optimization scheme, in which a metaheuristic method controls the discrete design variables and a gradient-based method controls the continuous design variables, combining the strengths of the two methods: the ability to handle discrete variables and fast convergence in terms of the continuous variables. The hybrid method is applied to two realistic steel structures: a multistory multibay braced frame and the roof structure of the Market Hall in Ghent, Belgium. The test problems take into account all relevant constraints in Eurocode 3 (design rules for steel structures): strength and stability constraints in the ultimate limit state and deformation constraints in the serviceability limit state. The test structures are composed of standard European I beams (IPE), European wide flange beams (HE), square hollow sections (SHS) and full round sections (R). The MATLAB source code for the test problems is made available on the Engineering Structures website.

This article is organized as follows. Section 2 discusses the concept of the hybrid algorithm in further detail, Section 3 presents modifications to make the design rules of Eurocode 3 suitable for gradient-based optimization, Section 4 gives an overview of the test problems, Section 5 presents the results of the numerical tests, and Section 6 gives final conclusions.

\section{Hybrid optimization scheme}

Three strategies can be distinguished in structural optimization: size, shape and topology optimization. In some problems, multiple strategies are combined, resulting in a mixture of discrete and continuous design variables, making them more difficult to solve. Studies that combine all three strategies are scarce and often rely on metaheuristic methods (e.g. Rajan [33], Balling et al. [34], Martini [35], Mortazavi and Toğan [36]), even though the academic community agrees that, for continuous design variables, gradient-based methods are much more effective [37]. With this in mind, a new hybrid optimization scheme is presented that combines metaheuristic and gradient-based methods following a nested approach, consisting of an inner and an outer loop. In the inner loop, the continuous design variables $\mathbf{x}_{\mathrm{c}}$ are optimized by a gradient-based algorithm, 


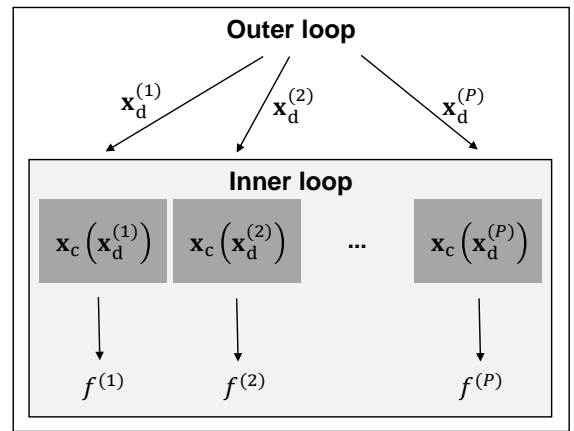

Metaheuristic algorithm generates $P$ sets of discrete design variables $\mathbf{x}_{\mathrm{d}}$

Gradient-based algorithm computes optimal values for the continuous design variables $\mathbf{x}_{\mathrm{c}}\left(\mathbf{x}_{\mathrm{d}}\right)$

The resulting objective function values are computed and used to generate the next iteration

\section{1 iteration}

Figure 1: Overview of the hybrid optimization scheme. Discrete design variables are optimized by a metaheuristic outer loop and continuous variables by a gradient-based inner loop.

while in the outer loop the discrete design variables $\mathbf{x}_{\mathrm{d}}$ are controlled by a metaheuristic algorithm (Figure 1). In this way, the optimization scheme restricts the search space of the metaheuristic algorithm to the discrete design variables while guaranteeing (local) optimality for the associated continuous design variables. This approach is conceptually similar to the implicit programming technique as e.g. in Achtziger [38].

\subsection{Formulation of the optimization problem}

The hybrid optimization scheme is intended for optimization problems of the following general form:

$$
\begin{aligned}
\min _{\mathbf{x}_{\mathrm{c}}, \mathbf{x}_{\mathrm{d}}} & f_{0}\left(\mathbf{x}_{\mathrm{c}}, \mathbf{x}_{\mathrm{d}}\right) \\
\text { subject to } & f_{i}\left(\mathbf{x}_{\mathrm{c}}, \mathbf{x}_{\mathrm{d}}\right) \leq 0, \quad i=1, \ldots, p
\end{aligned}
$$

where the objective $f_{0}\left(\mathbf{x}_{\mathrm{c}}, \mathbf{x}_{\mathrm{d}}\right)$ is a function of $n_{\mathrm{c}}$ continuous design variables $\mathbf{x}_{\mathrm{c}} \in \mathbb{R}^{n_{\mathrm{c}}}$ and $n_{\mathrm{d}}$ discrete design variables $\mathbf{x}_{\mathrm{d}} \in \mathbb{R}^{n_{\mathrm{d}}}$. The problem is subject to $p$ inequality constraint functions $f_{i}\left(\mathbf{x}_{\mathrm{c}}, \mathbf{x}_{\mathrm{d}}\right)$.

\subsection{Gradient-based inner loop}

The optimization scheme proceeds as follows. Associated with each set of discrete variables $\mathbf{x}_{\mathrm{d}}$ that is generated by the outer loop (as will be explained later), a gradient-based algorithm computes optimal values for the continuous design variables $\mathbf{x}_{\mathrm{c}}\left(\mathbf{x}_{\mathrm{d}}\right)$. To this end, the gradient-based algorithm solves the following continuous optimization sub-problem:

$$
\begin{aligned}
\mathbf{x}_{\mathrm{c}}\left(\mathbf{x}_{\mathrm{d}}\right)=\underset{\mathbf{x}_{\mathrm{c}}}{\arg \min } & f_{0}\left(\mathbf{x}_{c}, \mathbf{x}_{\mathrm{d}}\right) \\
\text { subject to } & f_{i}\left(\mathbf{x}_{\mathrm{c}}, \mathbf{x}_{\mathrm{d}}\right) \leq 0,
\end{aligned}
$$


where the objective $f_{0}$ and the constraints $f_{i}$ are continuously differentiable functions.

Because the discrete design variables $\mathbf{x}_{\mathrm{d}}$ can make the problem infeasible, a so-called 'fast feasibility check' has been developed, in which the inner loop workflow is divided into two phases. In order to quickly check the existence of a feasible solution, in the first phase the gradient of the objective function $f_{0}$ is artificially set to zero, which makes solving the optimization sub-problem equivalent to minimizing (the violation of) the constraints. If no feasible point can be found, the inner loop algorithm is terminated. If a feasible point is found, phase 2 is initiated and the sub-problem is solved again with the correct gradient information, starting from the solution of phase 1.

\subsection{Metaheuristic outer loop}

In the outer loop, a metaheuristic algorithm generates $P$ sets of discrete design variables $\mathbf{x}_{\mathrm{d}} \cdot{ }^{1}$ Associated with each set $\mathbf{x}_{\mathrm{d}}$, the gradient-based algorithm in the inner loop computes optimal values for the continuous design variables $\mathbf{x}_{\mathrm{c}}\left(\mathbf{x}_{\mathrm{d}}\right)$, following the procedure described in the previous subsection. The resulting objective and constraint function values are used to compute a modified objective function value $f$ for the original optimization problem (Equation (1)) as follows:

$$
f=f_{0}\left(\mathbf{x}_{\mathrm{c}}\left(\mathbf{x}_{\mathrm{d}}\right), \mathbf{x}_{\mathrm{d}}\right)\left(1+\kappa \sum_{i=1}^{p} \max \left(0, f_{i}\left(\mathbf{x}_{\mathrm{c}}\left(\mathbf{x}_{\mathrm{d}}\right), \mathbf{x}_{\mathrm{d}}\right)\right)\right)
$$

where $\kappa$ is a penalty coefficient, as in the work by Rajeev and Krishnamoorthy [39]. It is assumed that $f_{0}\left(\mathbf{x}_{\mathrm{c}}\left(\mathbf{x}_{\mathrm{d}}\right), \mathbf{x}_{\mathrm{d}}\right)$ is strictly positive, as is the case for the problems covered in this paper (weight minimization problems with appropriate bounds on the design variables). ${ }^{2}$ The modified objective function transforms the original problem into an unconstrained optimization problem that can be solved by any metaheuristic method.

\section{Eurocode design}

This section covers the design rules relevant for the test problems considered in the following section. These are based on EN 1993-1-1 (2015), the general rules for the design of steel structures (abbreviated as EC3), and EN 1993-1-1 ANB:2018, the Belgian National Annex to Eurocode 3 (abbreviated as ANB). The section consists of two subsections. The first subsection introduces the applicable clauses of Eurocode 3. The second subsection discusses the modifications needed to make these design rules suitable for gradient-based optimization. Readers familiar with the Eurocode may skip Section 3.1 and immediately proceed to Section 3.2 .

\footnotetext{
${ }^{1}$ The value of $P$ is equal to 1 for trajectory based metaheuristic methods and equal to the population size for population-based metaheuristic methods.

${ }^{2}$ For problems where the objective function may become negative, another penalty function is needed in order to prevent incorrectly endorsing infeasible designs.
} 


\subsection{Relevant design rules}

The design rules in Eurocode 3 consist of a number of verifications (checks) to be fulfilled at each point of the structure for the relevant design load combinations. Since it is impractical to monitor every point of the structure, this study considers five interpolation points per structural member in which the verifications are performed. The outcomes are expressed as a utilization ratios (UR), computed as actual values divided by maximum allowable values. For example, the variable $\mathrm{UR}_{\mathrm{R}, c, e p l}$ represents the utilization ratio for the resistance $(\mathrm{R})$, evaluated for check $c$ in member $e$, interpolation point $p$ and load combination $l$. Analogously, $\mathrm{UR}_{\mathrm{S}, c, e p l}$ represents the utilization ratios for stability (S) and $\mathrm{UR}_{\delta, p l}$ the utilization ratios for deformations $(\delta)$.

\subsubsection{Global analysis}

The internal forces and displacements in the structures are obtained from linear-elastic first-order analysis. The effects of initial sway imperfections are disregarded, assuming that the braced frame test problem is non-sway and that the design wind load on the Market Hall problem makes the impact of sway imperfections insignificant [EC3: §5.3.2(4)B]. The dynamic response of the structure, failure of the foundations, and fatigue are not taken into account.

\subsubsection{Ultimate Limit State}

The Ultimate Limit State (ULS) verifications check whether a structure is safe under the design load. Material imperfections are taken into account by means of a reduced yield strength for cross-sections with a nominal (flange) thickness of more than $40 \mathrm{~mm}$, based on [ANB: Tables $3.3 \& 3.4$ ], except for full round $\mathrm{R}$ sections to which no reduction is applied.

The cross-sections of the members are classified into four classes prior to optimization according to [EC3: §5.5], where the highest class of the compressed parts determines the class of the entire cross-section. The classification is performed separately for uniform compression and uni-axial bending about both principal axes. The resistance to individual actions is computed on the basis of the class for the relevant action, while the resistance to combined actions is determined by the highest class in bending.

The expressions to calculate the utilization ratios $U R_{R}$ and $U R_{S}$ for the resistance of cross-sections and the stability of the structural members are described in detail in Appendix A. All utilization ratios are evaluated in five interpolation points, spaced evenly along the longitudinal axis of the members.

\subsubsection{Serviceability limit state}

The Serviceability Limit State (SLS) verifications check whether the deformations of a structure remain within acceptable limits. The corresponding utilization ratios are computed as follows:

$$
\mathrm{UR}_{\delta, c, e p l}=\frac{\left|\delta_{\mathrm{Ed}}\right|}{\delta_{\lim }}
$$


where $\delta_{\mathrm{Ed}}$ is the design deformation value, computed from the combinations of actions in SLS, and $\delta_{\lim }$ the associated limit value. A distinction is made between individual member deflections (local deformations) and deformations of the overall structure (global deformations). The specific limit values are defined for each test problem separately in Section 4.

\subsection{Modifications for gradient-based optimization}

The design rules from Eurocode 3 are reformulated as constraint functions suitable for gradient-based shape optimization. The resulting constraint functions should (1) be continuously differentiable and (2) accurately reflect the degree of (in)feasibility of the structure. Furthermore, the constraint functions should be equal to or more conservative than the actual Eurocode constraints. The following four key principles apply:

1. The definition of the constraints as utilization ratios is preserved in order to give equal importance to different types of constraints.

2. Constraints that include absolute values are replaced by an equivalent pair of constraints, one assuming a positive and the other assuming a negative value.

3. The minimum and maximum functions are replaced by smooth approximations if this results in a conservative approach.

4. In the few cases where principles 2 and 3 cannot be applied, the Eurocode clauses are omitted or simplified. The corresponding modifications are given in Section 3.2.3.

The last three key principles are discussed in more detail in the following paragraphs.

\subsubsection{Absolute value function}

Many Eurocode checks are based on the absolute value of a force $F_{\mathrm{Ed}}$, which is not differentiable in the point $F_{\mathrm{Ed}}=0$. Consequently, constraints that involve one or more absolute value operations are broken down into sets of equivalent constraints. For example, the shear capacity $\mathrm{UR}_{\mathrm{R}, \mathrm{V}, i, e p l}$ in Equation (A.2) is replaced by $\mathrm{UR}_{\mathrm{R}, \mathrm{V}, i, e p l}^{(s)}(s=1,2)$, with:

$$
\begin{aligned}
\mathrm{UR}_{\mathrm{R}, \mathrm{V}, i, e p l}^{(1)} & =\frac{V_{i, \mathrm{Ed}}}{V_{\mathrm{pl}, i, \mathrm{Rd}}}, \\
\mathrm{UR}_{\mathrm{R}, \mathrm{V}, i, e p l}^{(2)} & =-\frac{V_{i, \mathrm{Ed}}}{V_{\mathrm{pl}, i, \mathrm{Rd}}} .
\end{aligned}
$$


Similarly, the utilization ratio $\mathrm{UR}_{\mathrm{R}, \mathrm{C}, 1,2, e p l}$ in Equation (A.13) is replaced by $\mathrm{UR}_{\mathrm{R}, \mathrm{C}, 1,2, e p l}^{(s)}(s=1,2,3,4)$ :

$$
\begin{aligned}
\mathrm{UR}_{\mathrm{R}, \mathrm{C}, 1,2, e p l}^{(1)} & =\Re\left(\left(\frac{M_{y, \mathrm{Ed}}}{M_{\mathrm{NV}, y, \mathrm{Rd}}}\right)^{\alpha}+\left(\frac{M_{z, \mathrm{Ed}}}{M_{\mathrm{NV}, z, \mathrm{Rd}}}\right)^{\beta}\right), \\
\mathrm{UR}_{\mathrm{R}, \mathrm{C}, 1,2, e p l}^{(2)} & =\Re\left(\left(\frac{M_{y, \mathrm{Ed}}}{M_{\mathrm{NV}, y, \mathrm{Rd}}}\right)^{\alpha}+\left(\frac{-M_{z, \mathrm{Ed}}}{M_{\mathrm{NV}, z, \mathrm{Rd}}}\right)^{\beta}\right), \\
\mathrm{UR}_{\mathrm{R}, \mathrm{C}, 1,2, e p l}^{(3)} & =\Re\left(\left(\frac{-M_{y, \mathrm{Ed}}}{M_{\mathrm{NV}, y, \mathrm{Rd}}}\right)^{\alpha}+\left(\frac{M_{z, \mathrm{Ed}}}{M_{\mathrm{NV}, z, \mathrm{Rd}}}\right)^{\beta}\right), \\
\mathrm{UR}_{\mathrm{R}, \mathrm{C}, 1,2, e p l}^{(4)} & =\Re\left(\left(\frac{-M_{y, \mathrm{Ed}}}{M_{\mathrm{NV}, y, \mathrm{Rd}}}\right)^{\alpha}+\left(\frac{-M_{z, \mathrm{Ed}}}{M_{\mathrm{NV}, z, \mathrm{Rd}}}\right)^{\beta}\right) .
\end{aligned}
$$

making use of the fact that for any two real numbers $a \geq 0, x \neq 0$, it holds that $a^{x}=\Re\left(a^{x}\right) \geq \Re\left((-a)^{x}\right)$, where $\Re$ denotes the real part. As a final example, the utilization ratios $\mathrm{UR}_{\mathrm{R}, \mathrm{C}, 3,4, e p l}$ in Equation (A.3) are replaced by $\mathrm{UR}_{\mathrm{R}, \mathrm{C}, 3,4, e p l}^{(s)}$ $(s=1, \ldots, 8)$ :

$$
\begin{aligned}
\mathrm{UR}_{\mathrm{R}, \mathrm{C}, 3,4, e p l}^{(1)} & =\frac{N_{\mathrm{Ed}}}{N_{\mathrm{t}, \mathrm{Rd}}}+\frac{M_{y, \mathrm{Ed}}}{M_{\mathrm{V}, y, \mathrm{Rd}}}+\frac{M_{z, \mathrm{Ed}}}{M_{\mathrm{V}, z, \mathrm{Rd}}}, \\
\mathrm{UR}_{\mathrm{R}, \mathrm{C}, 3,4, e p l}^{(2)} & =\frac{N_{\mathrm{Ed}}}{N_{\mathrm{t}, \mathrm{Rd}}}+\frac{M_{y, \mathrm{Ed}}}{M_{\mathrm{V}, y, \mathrm{Rd}}}-\frac{M_{z, \mathrm{Ed}}}{M_{\mathrm{V}, z, \mathrm{Rd}}}, \\
\mathrm{UR}_{\mathrm{R}, \mathrm{C}, 3,4, e p l}^{(3)} & =\frac{N_{\mathrm{Ed}}}{N_{\mathrm{t}, \mathrm{Rd}}}-\frac{M_{y, \mathrm{Ed}}}{M_{\mathrm{V}, y, \mathrm{Rd}}}+\frac{M_{z, \mathrm{Ed}}}{M_{\mathrm{V}, z, \mathrm{Rd}}}, \\
\mathrm{UR}_{\mathrm{R}, \mathrm{C}, 3,4, e p l}^{(4)} & =\frac{N_{\mathrm{Ed}}}{N_{\mathrm{t}, \mathrm{Rd}}}-\frac{M_{y, \mathrm{Ed}}}{M_{\mathrm{V}, y, \mathrm{Rd}}}-\frac{M_{z, \mathrm{Ed}}}{M_{\mathrm{V}, z, \mathrm{Rd}}}, \\
\mathrm{UR}_{\mathrm{R}, \mathrm{C}, 3,4, e p l}^{(5)} & =-\frac{N_{\mathrm{Ed}}}{N_{\mathrm{c}, \mathrm{Rd}}}+\frac{M_{y, \mathrm{Ed}}}{M_{\mathrm{V}, y, \mathrm{Rd}}}+\frac{M_{z, \mathrm{Ed}}}{M_{\mathrm{V}, z, \mathrm{Rd}}}, \\
\mathrm{UR}_{\mathrm{R}, \mathrm{C}, 3,4, e p l}^{(6)} & =-\frac{N_{\mathrm{Ed}}}{N_{\mathrm{c}, \mathrm{Rd}}}+\frac{M_{y, \mathrm{Ed}}}{M_{\mathrm{V}, y, \mathrm{Rd}}}-\frac{M_{z, \mathrm{Ed}}}{M_{\mathrm{V}, z, \mathrm{Rd}}}, \\
\mathrm{UR}_{\mathrm{R}, \mathrm{C}, 3,4, e p l}^{(7)} & =-\frac{N_{\mathrm{Ed}}}{N_{\mathrm{c}, \mathrm{Rd}}}-\frac{M_{y, \mathrm{Ed}}}{M_{\mathrm{V}, y, \mathrm{Rd}}}+\frac{M_{z, \mathrm{Ed}}}{M_{\mathrm{V}, z, \mathrm{Rd}}}, \\
\mathrm{UR}_{\mathrm{R}, \mathrm{C}, 3,4, e p l}^{(8)} & =-\frac{N_{\mathrm{Ed}}}{N_{\mathrm{c}, \mathrm{Rd}}}-\frac{M_{y, \mathrm{Ed}}}{M_{\mathrm{V}, y, \mathrm{Rd}}}-\frac{M_{z, \mathrm{Ed}}}{M_{\mathrm{V}, z, \mathrm{Rd}}} .
\end{aligned}
$$

The absolute value operations in the other constraints are reformulated in a similar way. 


\subsubsection{Minimum and maximum functions}

Minimum and maximum functions are replaced by smooth approximations based on the Kreisselmeier-Steinhauser function [40]:

$$
Q_{k}\left(y_{1}, \ldots, y_{m}\right)=\frac{1}{k} \log \left(\sum_{i=1}^{m} e^{k y_{i}}\right)
$$

with $m$ input arguments and $k$ a parameter that governs the 'smoothness' of the approximation. For $k>0, Q_{k}$ becomes asymptotically equal to the true maximum function as $k \rightarrow \infty$. The function $Q_{k}$ is infinitely differentiable (except in the limit) and always gives an overestimation of the true maximum, with approximation error $\epsilon \leq \log (m) / k$. For $k<0, Q_{k}$ approximates the minimum function, with properties similar to those mentioned before (giving an underestimation of the true minimum). For the sake of clarity, we use $Q_{\max , k}$ to denote the case where $k>0$ (softmax) and $Q_{\min ,-k}$ to denote the case where $k<0$ (softmin).

Some examples are given to illustrate the approach. The reduction factors $r_{1}$ and $r_{2}$ in Equations (A.11) and (A.12) are restated as

$$
\begin{aligned}
& r_{1}=\frac{1-Q_{\max , 20}(-n, 0.5 a, n)}{1-0.5 a} \\
& r_{2}=1-\left(\frac{Q_{\max , 20}(-n, a, n)-a}{1-a}\right)^{2}
\end{aligned}
$$

where $n=N_{\mathrm{Ed}} / N_{\mathrm{pl}, \mathrm{Rd}}$ (without the absolute value). In these expressions, replacing the true maximum with the softmax function is conservative (see Figure 2). This is not the case for the equivalent uniform moment factor $C_{1}$ in Equation (A.22), approximated using the expression below:

$$
C_{1}=Q_{\max , 100}\left(\sqrt{\frac{35 M_{y, j}^{2}}{M_{y, j}^{2}+9 M_{y, 2}^{2}+16 M_{y, 3}^{2}+9 M_{y, 4}^{2}}}\right)-\epsilon,
$$

where it is understood that the softmax function has 5 input arguments, one for each value of $j$, with $M_{y, j}(j=1, \ldots, 5)$ the bending moment value about the strong axis in the $j$-th interpolation point. Here, the $Q_{\max }$ function may (unsafely) overestimate the value of $C_{1}$, which is avoided by subtracting the maximum approximation error $\epsilon=\log (5) / 100$ from the result. Similarly, the upper bound on $\lambda_{i}$ in Equations (A.30) to (A.37) is replaced by $\bar{\lambda}_{i}=Q_{\min , 100}\left(\lambda_{i}, 1\right)+\epsilon$, with $\epsilon=\log (2) / 100$. The maximum bending moment value in Equations (A.28) and (A.29) is approximated as

$$
\bar{M}_{i, \mathrm{Ed}}=Q_{\max , 200 / M_{i, \mathrm{Rd}}}\left(M_{i, 1}, M_{i, 2}, M_{i, 3}, M_{i, 4}, M_{i, 5}\right)
$$

when $\bar{M}_{i, E d}$ has a positive sign, and

$$
\bar{M}_{i, \mathrm{Ed}}=Q_{\min , 200 / M_{i, \mathrm{Rd}}}\left(M_{i, 1}, M_{i, 2}, M_{i, 3}, M_{i, 4}, M_{i, 5}\right)
$$


when $\bar{M}_{i \text { Ed }}$ has a negative sign, giving an error never greater than $\log (5) / 200=$ 0.008 times the bending resistance $M_{i \text {, Rd }}$ of the member at hand. Modifications to the other $\min / \max$ operations are straightforward. A complete overview is given in Table 1, together with the worst-case approximation error associated with each variable. The values for the smoothness parameter $k$ were determined based on a visual assessment of the approximations to the exact functions (see for example Figures 2, 3 and 4) and remain constant during optimization.
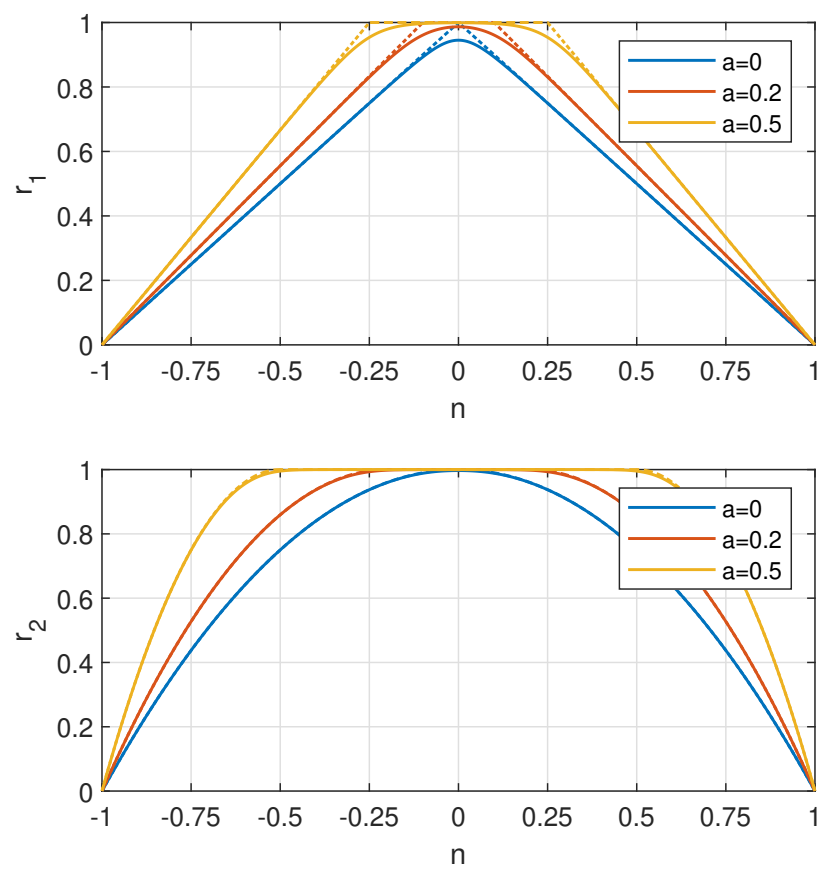

Figure 2: Reduction factors $r_{1}$ and $r_{2}$ for the effect of the axial force on the bending resistance (dashed lines) and their smooth approximations (solid lines) - Class 1 \& 2 cross-sections only.

\subsubsection{Additional simplifications}

A small number of constraints cannot be reformulated on the basis of the above principles and is therefore simplified. The equivalent uniform moment factors $C_{\mathrm{m}}$ in Table A.6 (on page 32 ) may be conservatively equated to 1 , although this approach is very conservative if (the absolute value of) the bending moment attains its largest value at one of the member ends. Therefore, the following approximation is used:

$$
C_{\mathrm{m}}=Q_{\mathrm{min}, 100}\left(C_{\mathrm{m}, 1}, C_{\mathrm{m}, 5}, 1\right)+\epsilon,
$$


Table 1: Overview of minimum/maximum functions and their smooth approximations. The last column shows the worst-case approximation error (within the feasible domain) for the variable in column 2

\begin{tabular}{lllcc}
\hline Eariable in column 2. & Variable & Variant of $Q_{k}$ & Correction & Max. error \\
\hline Eq. (A.5) & $\rho_{y}, \rho_{z}, \rho_{y z}$ & $Q_{\max , 100}$ & 0 & 0.000 \\
Eq. (A.11) & $r_{1}$ & $Q_{\max , 20}$ & 0 & 0.055 \\
Eq. (A.12) & $r_{2}$ & $Q_{\max , 20}$ & 0 & 0.007 \\
Eq. (A.16) & $\alpha, \beta$ & $Q_{\min , 20}$ & 0 & 0.668 \\
Eq. (A.18) & $\chi$ & $Q_{\min , 100}$ & 0 & 0.007 \\
Eq. (A.22) & $C_{1}$ & $Q_{\max , 100}$ & $-\log (5) / 100$ & 0.016 \\
Eq. (A.25) & $N_{c, \mathrm{Ed}}$ & $Q_{\min , 100}$ & 0 & 0.007 \\
Eq. (A.28), (A.29) & $\bar{M}_{i, \mathrm{Ed}}$ & $Q_{\min / \max , 200}$ & 0 & $0.008 \times M_{i, \mathrm{Rd}}$ \\
Eq. (A.30), (A.33), (A.34), (A.37) & $\bar{\lambda}_{i}$ & $Q_{\min , 100}$ & $+\log (2) / 100$ & 0.007 \\
Eq. (A.38), (A.39) & $k_{z y}$ & $Q_{\min , 20}$ & 0 & 0.023 \\
\hline
\end{tabular}
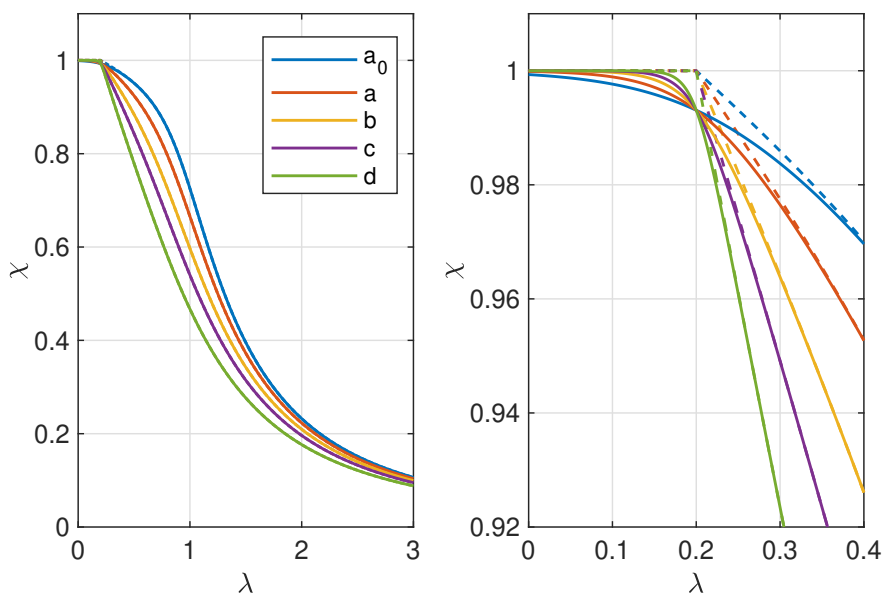

Figure 3: The difference between the true reduction factors $\chi$ (dashed lines) and their smooth minimum approximation (solid lines) is never more than 0.007 . The figure on the right shows a close-up of the targeted part of the domain.

with $\epsilon=\log (3) / 100=0.011$ and

$$
C_{\mathrm{m}, j}=Q_{\max , 100}\left(0.2-\frac{0.8 M_{i, 3}}{M_{i, j}}, 0.2+\frac{0.8 M_{i, 3}}{M_{i, j}}, 0.4\right),
$$

$(j=1,5)$ where $C_{\mathrm{m}}$ is either $C_{\mathrm{my}}, C_{\mathrm{mz}}$ or $C_{\mathrm{mLT}}$ and where $M_{i, 3}$ represents the bending moment value (about the relevant axis $i$ ) at the center and $M_{i, j}$ the moment at the member ends. In addition, the power $\beta$ in Equation (A.15) is conservatively set to 1 . Finally, all members are considered susceptible to torsional deformations unless $I_{\mathrm{T}} \geq I_{y}$, disregarding the condition in Equation (A.23). 


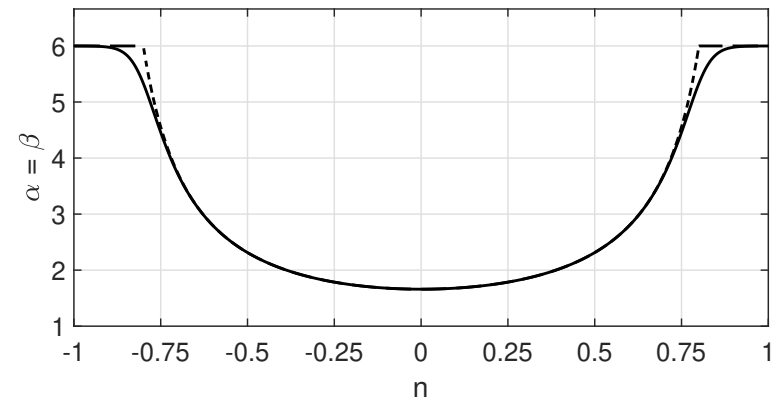

Figure 4: Powers $\alpha$ and $\beta$ for bi-axial bending of Class 1 and 2 SHS cross-sections (dashed line) and their smooth minimum approximation (solid line).

\section{Test problems}

Two realistic test problems are considered: a two-dimensional multistory multibay braced frame that is representative of many real-life structures (Figure 5) and the three-dimensional roof structure of the Market Hall in Ghent as a test problem with high architectural and urban relevance (Figure 6). The second problem (the Market Hall in Ghent) has been studied before from a size optimization perspective by Dillen et al. [41]. Both test problems involve a combination of continuous and discrete design variables. The analysis of the test problems is based on the finite element method [42] and its implementation in the Stabil toolbox for MATLAB / GNU Octave [43]. The source code is available on the Engineering Structures website. Isotropic linear-elastic material behavior is assumed in accordance with Hooke's law, where the material properties of steel are based on [EC3: §3.2.6] (modulus of elasticity $E=210 \mathrm{GPa}$ and Poisson's ratio $\nu=0.3$ ). The material density of steel is taken as $\rho=7851.8$ $\mathrm{kg} / \mathrm{m}^{3}$.

\subsection{Braced frame}

The braced frame structure is assumed to be part of an office building $17 \mathrm{~m}$ wide and $21 \mathrm{~m}$ high (Figure 5). The members of the structure are subdivided into 15 member groups (see Appendix B) for manufacturability reasons. The following design variables are considered:

- 15 discrete size variables $\mathbf{x}_{\mathrm{d} \text {,size }}$ that control the cross-sections of the structural members. The size variables are selected from $\mathcal{J}_{\mathrm{c}} \subset \mathbb{N}$, the index set of profiles in the section catalog $\mathcal{C}$ : each variable represents the index of a profile in the section catalog (see Appendix B), from which the corresponding section properties (see Appendix C) can be retrieved. The profiles of the floor and roof beams have to be selected from 23 IPE profiles, those of the columns from 24 HEA profiles, and those of the wind braces from 72 SHS sections. 
- 6 discrete topology variables $\mathbf{x}_{\mathrm{d} \text {,topology }}$ that control the presence and location of sway bracings on each floor. Each variable can have four values: 0 if there is no brace on the current floor, and 1, 2 or 3 for a brace on the current floor in the first, second and third bay, respectively.

- 4 continuous shape variables $\mathbf{x}_{\mathrm{c}, \text { shape }}$ that control the location of the columns. Each design variable represents the $\mathrm{x}$-coordinate of a single column (Figure 5).

The braced frame is assumed to be in the center of the office building and is subjected to 21 load cases according to Eurocode 1, including self-weight, permanent load, imposed load on the floors (category B: office buildings) and the roof (category H: inaccessible roofs), snow load and wind load (16 scenarios) based on the worst-case scenario for weather actions in Belgium (see Appendix D). The effect of the load cases is combined according to the procedure in (the Belgian National Annex to) Eurocode 0, with Consequences Class 2 and a design working life of 50 years, resulting in 1684 load combinations in the ultimate limit state and 71 load combinations in the serviceability limit state.
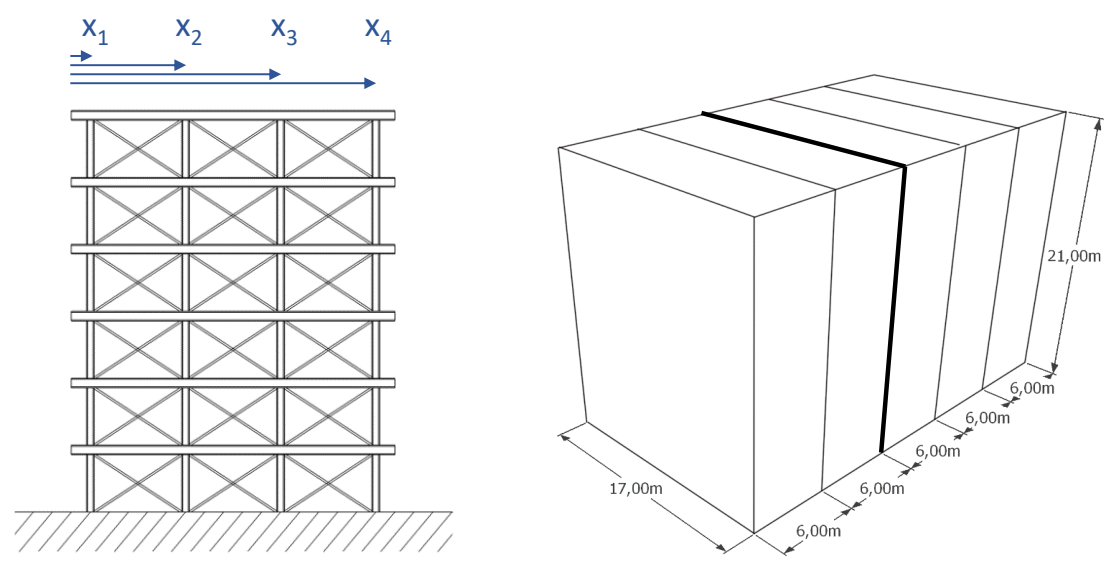

Figure 5: Braced frame: shape design variables and building characteristics.

The structure is subject to the Eurocode constraints from Section 3. The yield strength $f_{\mathrm{yk}}$ of the steel is selected according to the steel grade (S355). The deflections of the individual members are limited to $L / 300$ (or $L / 150$ for cantilever beams), in accordance with the Belgian standard NBN B03-003 (2003). ${ }^{3}$ Furthermore, the horizontal displacements between successive stories are limited to the height of a single story $(3.5 \mathrm{~m})$ divided by 250 . The buckling length

\footnotetext{
${ }^{3}$ Usually, the member deflection constraints are met when the resistance and stability constraints are satisfied and can therefore be omitted during optimization and checked afterwards.
} 
$L_{\mathrm{cr}}$ is generally taken as the system length (with reductions according to [EC3: $\S$ BB.1]), except for flexural buckling of the cantilever beams, where for all buckling modes the buckling length is taken as $L_{\mathrm{cr}}=2 L$. Finally, five additional linear inequality constraints ${ }^{4}$ ensure that the columns stay within the building envelope and do not swap.

\subsection{Market Hall}

The Market Hall in Ghent was designed by Robbrecht \& Daem and MarieJosé Van Hee Architects, with structural design by BAS and Stabilogics. The roof is $39 \mathrm{~m}$ long, $15 \mathrm{~m}$ wide, $16 \mathrm{~m}$ high and point-symmetrical around the center (Figure 6). It is designed as a reticulated folding structure, which spans in the longitudinal direction and is supported by four concrete abutments at the corners. The abutments are modelled as spring-loaded bearings with spring stiffness equal to $550 \times 10^{3} \mathrm{kN} / \mathrm{m}$ in the vertical direction. In the horizontal direction, the displacements at the supports are fixed, with the exception of the longitudinal displacements of the nodes facing the two chimneys. The roof planes are constructed as steel trusses in which diagonal tie rods provide in-plane stiffness. Three horizontal Vierendeel girders are installed on each of the side walls to absorb the wind load and thrust forces. The following design variables are considered:

- 13 discrete size variables $\mathbf{x}_{\mathrm{d} \text {,size }}$ that control the cross-sections of the structural members. The size variables are again selected from the index set $\mathcal{J}_{\mathrm{c}}$ of profiles in the section catalog $\mathcal{C}$ (see Appendix B). The profile of the tie rods has to be selected from 44 full round sections, the profiles of the main frame from $57 \mathrm{HE}$ type $\mathrm{A}, \mathrm{B}$ and $\mathrm{M}$ sections, and those of the remaining members from 48 SHS sections.

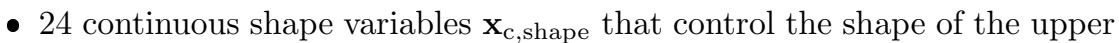
Vierendeel girders (making use of point-symmetry, see Figure 7).

The outer shape of the roof stems from the urban context rather than structural considerations and should therefore remain unaltered. The structural model of the Market Hall has been developed in close collaboration with the structural design office (BAS), using the same geometry, loads and boundary conditions as in the original model [44]. Non-linear analysis is avoided by modelling the tie rods (diagonals) as bar elements (most bars are loaded in tension in the relevant load combinations), where the stiffness of crossing bars in the central bay is halved to make the combined effect of one bar in tension and one bar in compression roughly equal to the effect of a single tie rod in tension. The impact of this simplification was found to be minimal.

The structure is subject to the Eurocode constraints from Section 3. The yield strength $f_{\mathrm{yk}}$ depends on the steel grade, which is S235, S355 and S460

\footnotetext{
${ }^{4}$ These constraints are passed on to the genetic algorithm as linear inequality constraints as well, exploiting special functionality ensuring that linear constraints are implicitly satisfied by newly generated designs.
} 


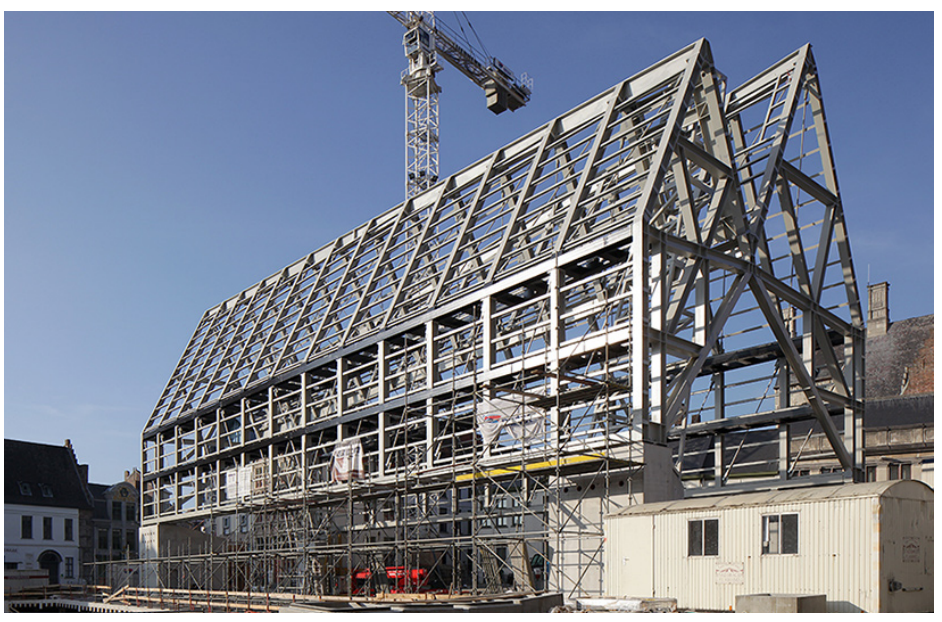

Figure 6: Roof structure of the Market Hall in Ghent [45].

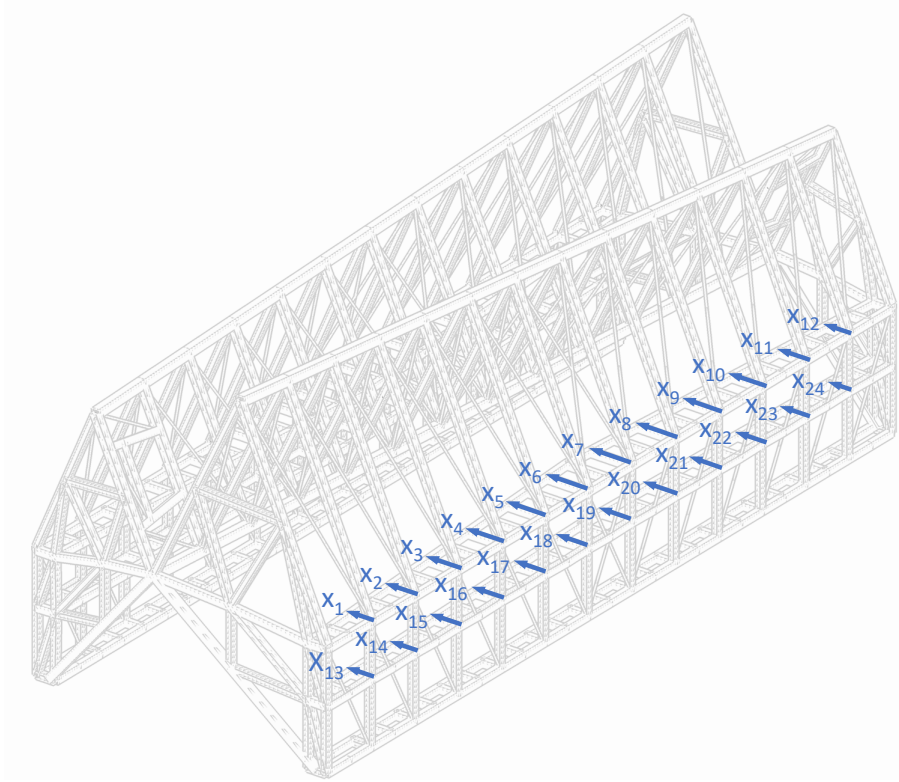

Figure 7: The shape design variables control the primary nodes of the Vierendeel girders. The coordinates of the intermediate secondary nodes are found by linear interpolation. The shape of the Vierendeel girders on the opposite side is obtained by point-symmetry.

for the HE, SHS and R sections, respectively. The deflection of the individual members should not exceed $L / 300$ (omitted during optimization and checked afterwards). For global deformations, separate limits have been defined for the 
total deformations $\delta_{\text {tot }}$ and for deformations caused by variable actions $\delta_{\text {var }}$, which are obtained by subtracting the effect of permanent actions (self-weight and permanent load) from the total actions. The deformations are evaluated at a number of critical points (Figure 8) and must not exceed pre-agreed values or the deformations of the original structure (where the pre-agreed values were exceeded, see Table 5 on page 25). Additional constraints are imposed that limit the side lengths of the cross-sections to $300 \mathrm{~mm}$ or to the side lengths of the corresponding cross-section in the original design - the larger of the two - in order to maintain an elegant structure. Similarly, the Vierendeel web members must not be larger than the chord members to avoid impractical joints. Two linear inequality constraints limit the area covered by the Vierendeel girders to that in the original design $\left(61.44 \mathrm{~m}^{2}\right.$ and $48.98 \mathrm{~m}^{2}$, respectively). Finally, box constraints limit the shape design variables to the interval between $0.35 \mathrm{~m}$ and $7.57 \mathrm{~m}$.

The buckling length $L_{\mathrm{cr}}$ of the members is generally taken as the system length (with reductions according to [EC3: §BB.1]), except for flexural buckling of the ridge and gutter beams (13 $\mathrm{m}$, based on a previous study), and out-ofplane buckling of the Vierendeel girder web $(2 L)$ and inner chord members $\left(L_{\text {system }} / 2\right)$, where $L_{\text {system }}$ represents the system length.

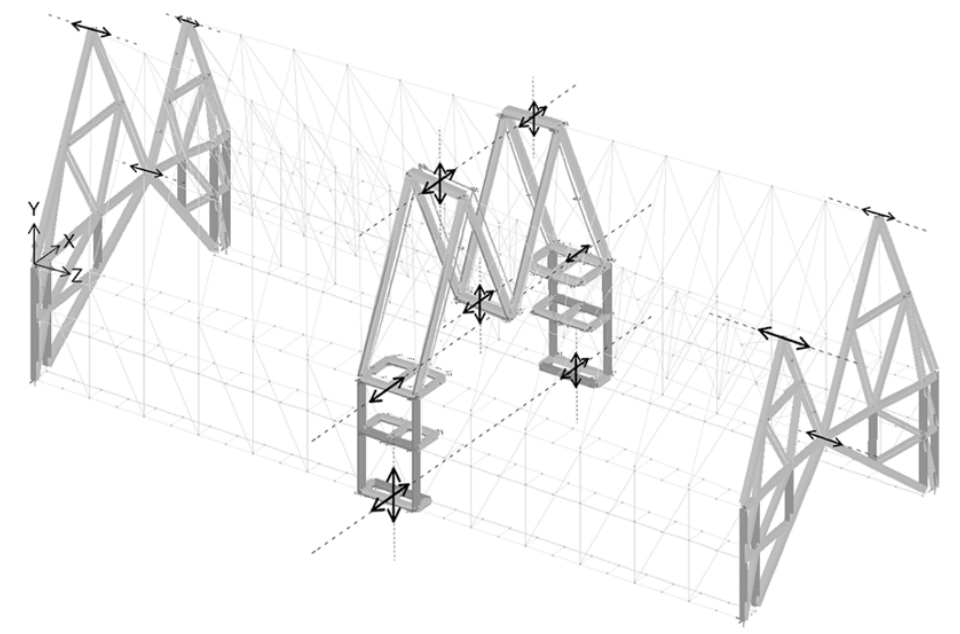

Figure 8: Verification of global deformations in SLS: critical points (figure source: Mertens [44] p.33)

\section{Results and discussion}

The hybrid optimization scheme presented in Section 2 is applied to the test problems in Section 4, taking into account the constraints in Section 3. The objective of the optimization problem is to minimize the weight of the 
steel structure while complying with the applicable Eurocode constraints. The problem is formulated as follows:

$$
\begin{array}{rl}
\min _{\mathbf{x}_{\mathrm{c}}, \mathbf{x}_{\mathrm{d}}} & W\left(\mathbf{x}_{\mathrm{c}}, \mathbf{x}_{\mathrm{d}}\right)=\rho \sum_{e=1}^{N} A_{e}\left(\mathbf{x}_{\mathrm{d}}\right) L_{e}\left(\mathbf{x}_{\mathrm{c}}\right) \\
\text { subject to } & \mathrm{UR}_{\mathrm{R}, c, e p l}\left(\mathbf{x}_{\mathrm{c}}, \mathbf{x}_{\mathrm{d}}\right)-0.9 \leq 0 \\
& \mathrm{UR}_{\mathrm{S}, c, e p l}\left(\mathbf{x}_{\mathrm{c}}, \mathbf{x}_{\mathrm{d}}\right)-0.9 \leq 0 \\
& \mathrm{UR}_{\delta, p l}\left(\mathbf{x}_{\mathrm{c}}, \mathbf{x}_{\mathrm{d}}\right)-1 \leq 0 \\
& x_{\mathrm{c}, \text { shape }, i}^{\min } \leq x_{\mathrm{c}, \text { shape }, i} \leq x_{\mathrm{c}, \text { shape }, i}^{\max } \\
& x_{\mathrm{d}, \text { size }, j} \in \mathcal{J}_{\mathrm{c}} \\
& x_{\mathrm{d}, \text { topology }, k} \in\{0,1,2,3\},
\end{array}
$$

where $\rho$ is the material density, $N$ the number of structural members, and $A_{e}$, $L_{e}$ the cross-sectional area and the length of member $e$. The terms $\mathrm{UR}_{\mathrm{R}, c, e p l}$, $\mathrm{UR}_{\mathrm{S}, c, e p l}$ and $\mathrm{UR}_{\delta, p l}$ denote the utilization ratios for the strength, stability and serviceability checks in the Eurocodes. A maximum utilization ratio of 0.9 is adopted for the capacity in the ultimate limit state to accommodate stress concentrations in the joints.

As described in Section 2.1, the continuous design variables $\mathbf{x}_{\mathrm{c}}$ are controlled by the inner loop and the discrete design variables $\mathbf{x}_{\mathrm{d}}$ by the outer loop of the hybrid algorithm. A modified objective function value $W_{\mathrm{p}}$ is computed, similar to $f$ in Equation (3) on page 6, as follows:

$$
W_{\mathrm{p}}=W\left(\mathbf{x}_{\mathrm{c}}, \mathbf{x}_{\mathrm{d}}\right)\left(1+\kappa_{\mathrm{R}} C_{\mathrm{R}}+\kappa_{\mathrm{S}} C_{\mathrm{S}}+\kappa_{\delta} C_{\delta}\right)
$$

with:

$$
\begin{aligned}
C_{\mathrm{R}} & =\sum_{e} \max \left(0, \max _{c, p, l}\left(\mathrm{UR}_{\mathrm{R}, c, e p l}\left(\mathbf{x}_{\mathrm{c}}, \mathbf{x}_{\mathrm{d}}\right)\right)-0.9\right) \\
C_{\mathrm{S}} & =\sum_{e} \max \left(0, \max _{c, p, l}\left(\mathrm{UR}_{\mathrm{S}, c, e p l}\left(\mathbf{x}_{\mathrm{c}}, \mathbf{x}_{\mathrm{d}}\right)\right)-0.9\right) \\
C_{\delta} & =\sum_{p} \max \left(0, \max _{l}\left(\mathrm{UR}_{\delta, p l}\left(\mathbf{x}_{\mathrm{c}}, \mathbf{x}_{\mathrm{d}}\right)\right)-1\right) .
\end{aligned}
$$

The coefficients $C_{\mathrm{R}}, C_{\mathrm{S}}$ and $C_{\delta}$ aggregate the constraints based on the highest UR-value along each member, summed over all members and all Eurocode checks. The coefficients $\kappa_{\mathrm{R}}, \kappa_{\mathrm{S}}$ and $\kappa_{\delta}$ are penalty coefficients similar to those in Rajeev and Krishnamoorthy [39], which can be used to balance the contributions of the strength, stability and deformation constraints, if this would be necessary.

The number of constraints in the optimization problem is huge. For example, for the Market Hall problem, more than $12 \times 10^{6}$ Eurocode constraints (after reformulation) must be evaluated. For each of these constraints, sensitivities must be computed with respect to 24 continuous shape design variables. The multitude of constraints makes iterative optimization solvers slow and memory intensive. This obstacle is inherent to Eurocode-constrained optimization and 
cannot be completely avoided, although two time-saving strategies have been implemented. These are discussed in Subsections 5.1 and 5.2. After that, the procedure for sensitivity analysis is discussed, the design of the tests is given and the hybrid optimization scheme is applied to the test problems.

\subsection{Optimization history log file}

A metaheuristic algorithm may generate the same combination of design variables multiple times during optimization. To avoid repeated solutions of the same sub-problem, the solutions of all evaluated designs are cached in a $\log$ file. When a set of discrete design variables is repeated, the corresponding objective function value is simply recovered from cache.

\subsection{Critical set strategy}

The Eurocode specifies a large number of design load scenarios, of which only a few will ultimately be normative. To improve computational efficiency and reduce memory requirements, the gradient-based algorithm in the inner loop works with a critical set of constraints, limited to those corresponding to the most restrictive load combinations and/or structural members. The size of the critical set is controlled by the critical fraction, which represents the percentage of load combinations / structural members included in the critical set. Initially, the critical fraction is set at $1 \%$ and the critical set is generated on the basis of a (ideally, but not necessarily) representative initial design, after which a number of successive gradient-based optimization runs are performed in which the critical set is systematically updated until a feasible design is obtained. When not all constraints are met, the optimization algorithm is restarted from the current solution. This approach is conceptually similar to that in Verbart and Stolpe [46].

\subsection{Sensitivity analysis}

Gradient-based algorithms use the sensitivities (gradients) of the objective and constraint functions to steer the search towards well-performing designs. The sensitivities $\partial \mathbf{U} / \partial x_{\mathrm{c}, i}$ of the nodal displacement matrix $\mathbf{U}$ with respect to the (continuous) design variables $x_{\mathrm{c}, i}$ are obtained via direct differentiation [47], based on the analytical sensitivities $\partial \mathbf{K} / \partial x_{\mathrm{c}, i}$ and $\partial \mathbf{P} / \partial x_{\mathrm{c}, i}$ of the stiffness matrix $\mathbf{K}$ and the external load matrix $\mathbf{P}$ obtained using Stabil [43]. The direct differentiation method (instead of the adjoint method) is used because the displacement sensitivities are directly required for the constraints in the serviceability limit state. The sensitivities $\partial \mathbf{U} / \partial x_{\mathrm{c}, i}$ of the nodal displacements are used to calculate sensitivities for the member forces, which in turn are used to calculate sensitivities for the Eurocode constraints using standard calculus. All sensitivities have been verified using finite differences. 


\subsection{Design of the tests}

Two numerical tests are performed, in which the genetic algorithm from the Global Optimization Toolbox in MATLAB (version R2019a) is used in the outer loop and the fmincon active-set SQP algorithm from the Optimization Toolbox in the inner loop. The control parameters are set to their default values, the tolerances on the objective function (FunctionTolerance and OptimalityTolerance) for the active-set SQP algorithm to $10^{-1}$, and the ConstraintTolerance and StepTolerance to $10^{-3}$. The penalty coefficients in Equation (28) are set to 10, as in Rajeev and Krishnamoorthy [39].

The hybrid algorithm is compared to the standard (conventional) genetic algorithm that comes with MATLAB version R2019a, controlling both the discrete $\mathbf{x}_{\mathrm{d}}$ and continuous design variables $\mathbf{x}_{\mathrm{c}} \cdot{ }^{5}$ The Eurocode modifications from Section 3.2 apply during gradient-based optimization only and do not apply to the conventional genetic algorithm or when generating or evaluating the critical set. In this sense, it is beneficial to combine the modified Eurocode constraints with the critical set strategy, as conservative approximations to the Eurocode do not affect the load combinations and structural members that do not belong to the critical set. The conventional genetic algorithm and the hybrid algorithm thus solve two slightly different problems, although the latter is always more conservative (according to the key principles in Section 3).

Two research questions arise: (1) does the hybrid genetic algorithm perform better than its conventional counterpart and (2) do the modifications needed to enable gradient-based optimization outweigh any potential efficiency gains? These research questions will be addressed in the remainder of this section.

\subsection{Braced frame test problem}

The braced frame problem is discussed first. The critical set strategy is employed to reduce the number of load combinations. The computations have been performed on a high performance computing cluster, using a single compute node with 192 GB RAM and 36 cores (Intel Skylake, 2.3 GHz). Within each generation, the objective function evaluations are divided over the available compute cores and evaluated simultaneously. The optimization is terminated after 6 hours (wall clock time, see Table 2). Both the conventional and the hybrid algorithm are restarted 10 times with random values for the discrete design variables $\mathbf{x}_{\mathrm{d}}$ and fixed values of $1,6,11$ and $16 \mathrm{~m}$ for the continuous design variables $\mathbf{x}_{\mathrm{c}}$.

\subsubsection{Results}

The sections of the members in the optimized designs (the best designs in the simulation) are listed in Table B.9 (in Appendix B). The convergence history is shown in Figure 9. The hybrid algorithm starts off slower (each fitness evaluation involves a complete inner loop optimization run rather than a single finite

\footnotetext{
${ }^{5}$ As with the log file strategy in Section 5.1, repeated function evaluations for the conventional genetic algorithm are prevented by recovering the solution from cache.
} 
Table 2: Computational times (in wall-clock time).

\begin{tabular}{ccc}
\hline & Genetic algorithm & Hybrid algorithm \\
\hline Braced frame problem & 6 hours & 6 hours \\
Market Hall problem & 12 hours & 12 hours \\
\hline
\end{tabular}

element analysis) but consistently converges to a solution that uses significantly less material than the solution found by the genetic algorithm, even though the latter algorithm is able to consider ten times more generations in the same time frame (Table 3). Comparable results are found for different optimization runs, but the hybrid algorithm does not guarantee finding the global optimum. With one exception, the hybrid algorithm consistently outperforms the conventional algorithm.

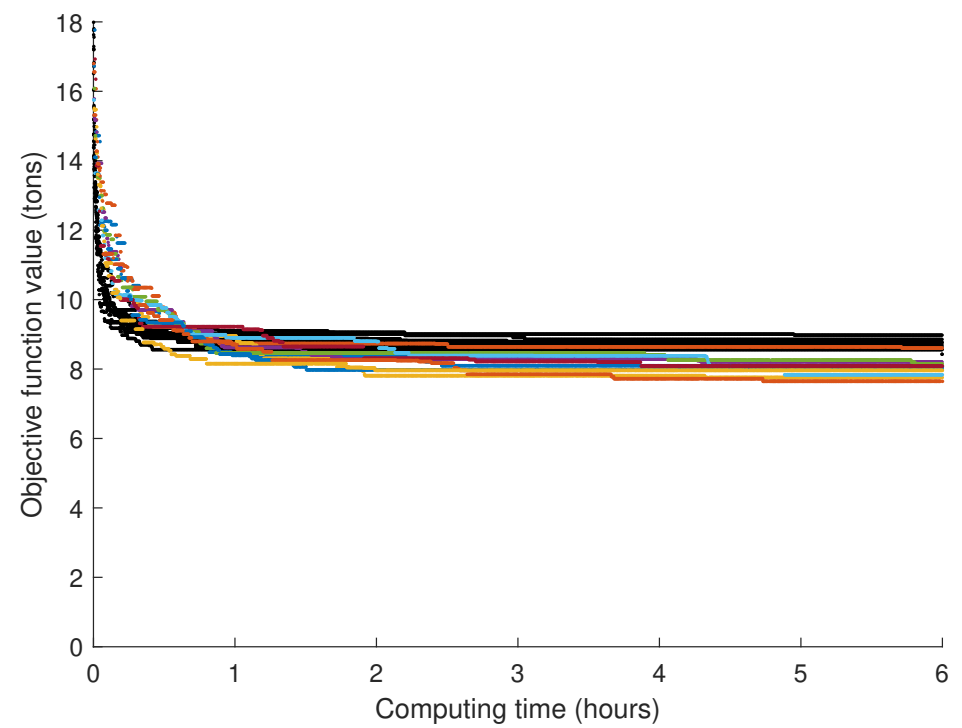

Figure 9: Convergence history for the braced frame. Each black dot represents the lowest objective function value $W_{\mathrm{p}}$ in one generation (100 individuals) of the genetic algorithm and each colored dot the lowest objective function value in one generation of the hybrid algorithm.

\subsubsection{Verification}

The geometry of the optimized designs is shown in Figures 10 and 11. The design by the conventional genetic algorithm (on the left) is almost $10 \%$ heavier than the design by the hybrid algorithm (on the right). Both designs are evaluated in Diamonds (a commercial finite element package that is popular among Belgian engineering firms) as a validation. The results are summarized in Figures 12 to 15. The maximum resistance utilization ratio $\mathrm{UR}_{\mathrm{R}}$ is $66.5 \%$ for the solution by the genetic algorithm and $79.2 \%$ for the solution by the hybrid 
Table 3: Test run history for the braced frame.

\begin{tabular}{cccc}
\hline \multicolumn{2}{c}{ Genetic algorithm } & \multicolumn{2}{c}{ Hybrid algorithm } \\
$W_{\mathrm{p}}$ value & \# generations & $W_{\mathrm{p}}$ value & \# generations \\
\hline 8.43 & 12755 & 7.65 & 1640 \\
8.56 & 13568 & 7.76 & 1826 \\
8.58 & 13375 & 7.84 & 1847 \\
8.58 & 12267 & 7.96 & 1849 \\
8.63 & 13115 & 7.98 & 1650 \\
8.69 & 12963 & 8.04 & 1508 \\
8.76 & 11871 & 8.09 & 1764 \\
8.77 & 12472 & 8.15 & 1702 \\
8.85 & 12066 & 8.21 & 1739 \\
8.98 & 12160 & 8.60 & 1801 \\
\hline
\end{tabular}

algorithm, which is well below the $90 \%$ limit value. The maximum stability utilization ratios $\mathrm{UR}_{\mathrm{S}}(90.9 \%$ and $90.7 \%)$ slightly exceed the limit value, which may be due to rounding errors (for the column coordinates) or small differences between the models in MATLAB and Diamonds. Given the complexity of the models, a difference of less than 1 percentage point is considered acceptable. The maximum $\mathrm{UR}_{\mathrm{S}}$ value for the conventional algorithm occurs in the third column on the third floor (G5) and the maximum $\mathrm{UR}_{\mathrm{S}}$ value for the hybrid algorithm is found in the roof beam (G2). Apart from these, the maximum $\mathrm{UR}_{\mathrm{S}}$ values are $89.6 \%$ and $89.2 \%$ for the design by the genetic and the hybrid algorithm, respectively.

All SLS constraints are comfortably met: the deflection of the floor and roof beams amounts to a maximum of $16.7 \%$ (conventional algorithm) and $34.3 \%$ (hybrid algorithm) of the limit value (considering variable loads only) and the interstory drift never exceeds $22.7 \%$ (conventional algorithm) and $22.5 \%$ (hybrid algorithm) of the limit value.

\subsubsection{Interpretation and discussion}

Table B.9 shows that, in both designs, the roof beam (G2) is lighter than the floor beams (G1). This is expected because the imposed load for offices floors exceeds the combination of maintenance and weather loads on the roof. In the design by the hybrid algorithm, the steel sections for the floor and roof beams are lighter than in the design by the genetic algorithm. The column members in G4 and G7 are slightly lighter in the design by the hybrid algorithm, while the reverse is true for the members in G6. The sections of the remaining members are identical. The design by the hybrid algorithm is more symmetrical (in terms of column placement) and the columns are more evenly spaced, resulting in more uniform utilization ratios along the floor and roof beams: aggregating all beam members into two member groups does not imply a major loss in structural efficiency. Additionally, the columns are positioned so that the length of a 
cantilever beam is equal to approximately 0.4 times the length of a spanning beam, which is in line with rules of thumb (for equality of end moments).

Even though the interstory drift constraints remain inactive, it appears appropriate to limit the lateral deflection of the frame in order to reduce bending in the structure. The optimizer provides sway braces on the lower three floors, using SHS $120 \times 5$ sections in both designs. This is the section with the smallest wall thickness (for the corresponding side length) in the section catalog, a feature common to the solutions of the other test runs (not shown here). The columns generally become smaller towards the top of the building, except for the central column members on the fourth floor (G6) in the design by the hybrid algorithm, where it was probably more economical to locally thicken the columns than to provide additional braces.

From the results it is concluded that the hybrid algorithm outperforms the genetic algorithm, even though the continuous design variables $\mathbf{x}_{\mathrm{c}}$ (the column coordinates) have little influence on the objective function $W$ (the amount of steel in the structure). The ability of the hybrid algorithm to specifically search for feasible designs for a given set of discrete design variables $\mathbf{x}_{\mathrm{d}}$ proves to be a major asset. This can be illustrated as follows: as the members of the braced frame become smaller, there will be fewer column coordinates for which all constraints are met. The genetic algorithm has to rely on coincidence to accidentally generate the right combination of member sections and column coordinates, whereas the hybrid algorithm can actively search for column coordinates that make the design feasible. In this example, the advantages of the hybrid strategy clearly outweigh the conservative Eurocode modifications needed to enable gradient based optimization.

\subsection{Market Hall test problem}

The Market Hall problem is considered next. The critical set strategy is employed to reduce the number of structural members. The computations have been performed on a high performance computing cluster, this time using a single compute node with 768 GB RAM and 36 cores (Intel Skylake, $2.3 \mathrm{GHz}$ ). The optimization is terminated after 12 hours (see Table 2).

\subsubsection{Results}

As before, a comparison is made between 10 independent runs of both algorithms (Figure 16), with random initial values for the discrete variables $\mathbf{x}_{\mathrm{d}}$ and fixed initial values of $0.94 \mathrm{~m}$ for the continuous variables $\mathbf{x}_{\mathrm{c}}$ - giving a rectangular shape to the Vierendeel girders. The (best) optimized designs are shown in Table B.10 (in Appendix B) and the test run history is shown in Table 4. The number of continuous design variables is larger than before (24 instead of 4) and the genetic algorithm finds widely different (and suboptimal) solutions, whereas the solutions produced by the hybrid algorithm are much closer together. With one exception, the hybrid algorithm always performs better than the genetic algorithm. The difference between the best results found by the two algorithms is $3.6 \%$ and the difference between the mean result is $19.6 \%$. 
Table 4: Test run history for the Market Hall.

\begin{tabular}{cccc}
\hline \multicolumn{2}{c}{ Genetic algorithm } & \multicolumn{2}{c}{ Hybrid algorithm } \\
$W_{\mathrm{p}}$ value & \# generations & $W_{\mathrm{p}}$ value & \# generations \\
\hline 191.39 & 7349 & 184.48 & 554 \\
200.76 & 7154 & 184.63 & 796 \\
204.41 & 7159 & 184.82 & 563 \\
208.07 & 6913 & 187.08 & 500 \\
209.64 & 6890 & 187.11 & 646 \\
246.17 & 7084 & 187.34 & 1105 \\
260.77 & 6763 & 188.61 & 762 \\
263.34 & 6722 & 188.67 & 1293 \\
269.37 & 7121 & 191.34 & 965 \\
281.09 & 6916 & 193.74 & 900 \\
\hline
\end{tabular}

\subsubsection{Verification}

Again, the optimized designs are validated in Diamonds (Figures 17 to 22). The largest resistance utilization ratio $\mathrm{UR}_{\mathrm{R}}$ for the design by the hybrid algorithm $(88.2 \%)$ is slightly higher than for the genetic algorithm $(84.0 \%)$. The resistance checks are generally not decisive, except for the members near the two trimmer constructions. Apart from the ridge beams, the gutter beam and the upper Vierendeel girder chords, roughly all $\mathrm{UR}_{R}$ values increase towards the supports. The maximum stability utilization ratio $\mathrm{UR}_{\mathrm{S}}$ is $92.1 \%$ for the design by the genetic algorithm and $89.3 \%$ for the design by the hybrid algorithm. The $90 \%$ limit is exceeded here because the buckling length of the inner chord of the lower Vierendeel girder computed by Diamonds $(21.6 \mathrm{~m})$ is slightly larger than the estimate of $19.6 \mathrm{~m}$ in MATLAB, which is probably related to the slender infill work in the front façade (G5) being unable to provide sufficient rotational support to the Vierendeel girder's outer ends to consider them stiff-jointed. Excluding this anomaly, the highest $\mathrm{UR}_{\mathrm{S}}$ value is $83.6 \%$, occurring in one of the rafters in the front façade.

As far as SLS is concerned, the deflections of the members are never more than $64.7 \%$ of their allowable value (for both algorithms). The global deformations are summarized in Table 5. For both optimized designs there is a small violation of the constraints in Diamonds (but not in MATLAB), again due to minor differences between the models.

Table B.10 shows the member sections of the original design and the optimized designs. It is observed that the side lengths of the cross-sections in the optimized designs never exceed those in the original design and that the side dimensions of the Vierendeel girder web members (G9) are not larger than the chord members (G7 \& G12) to which they are attached. The area covered by the upper and central Vierendeel girders is $61.44 \mathrm{~m}^{2}$ and $48.97 \mathrm{~m}^{2}$ for the design by the hybrid algorithm, which is almost the same as in the original design, compared to $60.97 \mathrm{~m}^{2}$ and $39.28 \mathrm{~m}^{2}$ for the design by the genetic algorithm. 
Table 5: Global SLS displacement values in Diamonds (in mm). Exclamation marks (!) are added next to the displacement values that exceed the limit value.

\begin{tabular}{|c|c|c|c|c|c|}
\hline Name & Description & Limit value & Original design & Genetic algorithm & Hybrid algorithm \\
\hline$\delta_{X, \lim , \mathrm{sb}, \mathrm{var}}$ & side walls bottom & 30.0 & 17.7 & 23.5 & 25.9 \\
\hline$\delta_{X, \lim , \mathrm{st}, \mathrm{var}}$ & side walls top & 36.7 & 36.7 & $36.8(!)$ & 36.6 \\
\hline$\delta_{X, \lim , \mathrm{rid}, \mathrm{var}}$ & ridge beams & 50.2 & 43.0 & 38.1 & 42.3 \\
\hline$\delta_{X, \lim , \text { rid,tot }}$ & ridge beams & 63.3 & 63.3 & 60.3 & $63.5(!)$ \\
\hline$\delta_{X, \text { lim,gut,var }}$ & gutter beam & 50.2 & 50.2 & 43.5 & 49.4 \\
\hline$\delta_{X, \lim , \text { gut,tot }}$ & gutter beam & 63.3 & 50.6 & 43.5 & 49.6 \\
\hline$\delta_{Y, \lim , \mathrm{var}}$ & mid-span & 30.0 & 9.9 & 9.6 & 10.1 \\
\hline$\delta_{Y, \lim , \text { tot }}$ & mid-span & 110.5 & 50.5 & 49.0 & 50.6 \\
\hline$\delta_{Z, \lim , \text { end,tot }}$ & front façades & 30.0 & 4.3 & 6.3 & 5.4 \\
\hline
\end{tabular}

\subsubsection{Interpretation and discussion}

The original design for the Market Hall weighs 226.6 tons. Both optimized designs perform better: the genetic algorithm uses 35 tons less steel $(15.5 \%)$, while the hybrid algorithm realizes material savings of more than 42 tons $(18.6 \%)$. The sections in the optimized designs are mostly lighter than in the original design, except for the tie rods (G1) and the rafters near the trimmer constructions (G8) in the design by the genetic algorithm. Important material savings are obtained in the ridge and gutter beams (G13, about 18 and 13 tons for the genetic and the hybrid algorithm, respectively), the chord members of the upper Vierendeel girders (G9, about 10 and 17 tons for genetic and hybrid) and the infill in the front façades (G4, about 10 tons for both).

Compared to the genetic algorithm, the hybrid algorithm mainly saves on material in the tie rods (G1) and the Vierendeel girders (G9). The figures show that the gradient-based algorithm created funicular shapes for the Vierendeel girders (which efficiently resist the horizontal load through axial forces instead of bending action), whereas the genetic algorithm failed to do so for the central Vierendeel girder. The shape of the Vierendeel girders in the design by the hybrid algorithm is asymmetrical: the structural height of the upper girder increases towards the high end of the roof, where the area affected by the wind load is larger. The central girder seems to compensate by making its shape slightly asymmetrical towards the other side. Again, the effectiveness of the hybrid optimization scheme outweighs the conservative approximations to the Eurocode.

\section{Conclusions}

This study addresses size, shape and topology optimization of steel structures, involving a combination of discrete (size and topology) and continuous (shape) design variables. In the literature, such problems are usually solved using metaheuristic methods, often genetic algorithms. Metaheuristic methods are universally applicable but relatively inefficient, and the consensus is that 
gradient-based optimization methods are much more effective in case the design variables are continuous. With this in mind, a hybrid optimization scheme is developed that combines the strengths of metaheuristic and gradient-based methods: the ability to handle discrete design variables and fast convergence in terms of continuous design variables.

The proposed optimization scheme is applied to two realistic steel structures: a fictitious multistory multibay braced frame and the roof structure of the Market Hall in Ghent. All applicable design rules from Eurocode 3 are taken into account, simulating a realistic design situation. The Eurocode constraints have been modified to make them suitable for gradient-based optimization (with respect to continuous shape design variables). Moreover, two time-saving strategies have been introduced that alleviate the computational burden typical of Eurocode-constrained design optimization.

The performance of the hybrid algorithm is compared with that of the genetic algorithm in MATLAB. For both test problems, the hybrid algorithm was able to improve the best solution of the genetic algorithm, despite several conservative approximations that had to be made when reformulating the Eurocode. Commercial finite element software was used to validate the results. The source code of the test problems has been made available on the Engineering Structures website to serve as benchmark problems for future development of structural optimization algorithms.

The present study is limited to linear-elastic first-order analysis of steel structures. The Eurocode reformulations in this paper will not be applicable to other building materials for which not all Eurocode constraints are differentiable. Future work will be required to efficiently address other building materials with the proposed optimization scheme. Independently, it might be possible to increase the efficiency of the hybrid algorithm using the upper bound strategy presented in Ref. [48], but this is beyond the scope of this paper.

\section{Acknowledgements}

This work has been performed in the frame of project C24/15/012 funded by the Industrial Research Fund of KU Leuven. The financial support is gratefully acknowledged. We would also like to thank H. Van Beurden and D. Jaspaert from BAS, D. Elleboog from BuildSoft support and Dr. E. Moravveji from the Flemish Supercomputer Center for their contributions to this work.

\section{A. Resistance \& stability checks in Eurocode 3}

\section{A.1. Resistance of cross-sections}

This section describes the utilization ratios $\mathrm{UR}_{\mathrm{R}}$ for the resistance of crosssections. The resistance to shear $V$ is discussed first. Next, the combination of axial force $N$, shear force $V$ and bending moment $M$ is discussed, separately 
for cross-sections of Class 3 / 4 and for cross-sections of Class $1 / 2$, followed by the resistance to torsion $T$. The design yield strength is taken as:

$$
f_{\mathrm{yd}}=\frac{f_{\mathrm{yk}}}{\gamma_{\mathrm{M} 0}}
$$

with $\gamma_{\mathrm{M} 0}=1$ (for buildings).

The utilization ratio for shear is as follows:

$$
\mathrm{UR}_{\mathrm{R}, \mathrm{V}, i, e p l}=\frac{\left|V_{i, \mathrm{Ed}}\right|}{V_{\mathrm{pl}, i, \mathrm{Rd}}}, \quad \text { with } V_{\mathrm{pl}, i, \mathrm{Rd}}=\frac{A_{\mathrm{v}, i} f_{\mathrm{yd}}}{\sqrt{3}}
$$

where $V_{i, \mathrm{Ed}}(i=y, z)$ is the design shear force and $A_{\mathrm{v}, i}$ the shear area.

The combined effect of shear, axial forces, and bi-axial bending for Class 3 and 4 cross-sections always outweighs the effects of each of these actions considered separately. The corresponding utilization ratio is:

$$
\mathrm{UR}_{\mathrm{R}, \mathrm{C}, 3,4, e p l}=\left\{\begin{aligned}
\frac{N_{\mathrm{Ed}}}{N_{\mathrm{t}, \mathrm{Rd}}}+\frac{\left|M_{y, \mathrm{Ed}}\right|}{M_{\mathrm{V}, y, \mathrm{Rd}}}+\frac{\left|M_{z, \mathrm{Ed}}\right|}{M_{\mathrm{V}, z, \mathrm{Rd}}} & \text { if } N_{\mathrm{Ed}} \geq 0 \\
-\frac{N_{\mathrm{Ed}}}{N_{\mathrm{c}, \mathrm{Rd}}}+\frac{\left|M_{y, \mathrm{Ed}}\right|}{M_{\mathrm{V}, y, \mathrm{Rd}}}+\frac{\left|M_{z, \mathrm{Ed}}\right|}{M_{\mathrm{V}, z, \mathrm{Rd}}} & \text { if } N_{\mathrm{Ed}}<0,
\end{aligned}\right.
$$

with $N_{\mathrm{t}, \mathrm{Rd}}=A f_{\mathrm{yd}}$ the design tension resistance, and $N_{\mathrm{c}, \mathrm{Rd}}=A f_{\mathrm{yd}}$ (Class 3 ) or $N_{\mathrm{c}, \mathrm{Rd}}=A_{\mathrm{eff}} f_{\mathrm{yd}}$ (Class 4$)$ the design compression resistance. ${ }^{6}$ In these expressions, $M_{\mathrm{V}, i, \mathrm{Rd}}$ is the design bending resistance, reduced due to the effect of shear [EC3: $§ 6.2 .9 .2(1) \mathrm{P}]$. It is computed as:

$$
M_{\mathrm{V}, i, \mathrm{Rd}}=W_{i}\left(1-\rho_{y z}\right) f_{\mathrm{yd}}
$$

where $W_{i}(i=y, z)$ is the elastic section modulus $W_{\mathrm{el}, i}$ for Class 3 cross-sections and the effective section modulus $W_{\text {eff }, i}$ for Class 4 cross-sections [EC3: Eq. 6.29]. The corresponding reduction factor $\rho_{y z}$ is given by:

$$
\rho_{y z}=\left[2 \max \left(\sqrt{\left(\frac{V_{y, \mathrm{Ed}}}{V_{\mathrm{pl}, y, \mathrm{Rd}}}\right)^{2}+\left(\frac{V_{z, \mathrm{Ed}}}{V_{\mathrm{pl}, z, \mathrm{Rd}}}\right)^{2}}, 0.5\right)-1\right]^{2} .
$$

For Class 1 and 2 cross-sections, the individual effects of axial force and bending must be considered separately, with

$$
\mathrm{UR}_{\mathrm{R}, \mathrm{N}, e p l}=\frac{\left|N_{\mathrm{Ed}}\right|}{N_{\mathrm{pl}, \mathrm{Rd}}}, \quad N_{\mathrm{pl}, \mathrm{Rd}}=A f_{\mathrm{yd}}
$$

the utilization ratio for the axial force, and

$$
\mathrm{UR}_{\mathrm{R}, \mathrm{M}, i, e p l}=\frac{\left|M_{i, \mathrm{Ed}}\right|}{M_{\mathrm{NV}, i, \mathrm{Rd}}}
$$

\footnotetext{
${ }^{6} \mathrm{By}$ convention, an axial force $N_{\mathrm{Ed}}$ is positive in tension and negative in compression.
} 
the utilization ratio for uni-axial bending. Herein, $M_{\mathrm{NV}, i, \mathrm{Rd}}$ is the reduced bending resistance due to both axial force and shear, computed as:

$$
\begin{aligned}
& M_{\mathrm{NV}, y, \mathrm{Rd}}=r_{1} W_{\mathrm{pl}, y}\left(1-\rho_{z}\right) f_{\mathrm{yd}}, \\
& M_{\mathrm{NV}, z, \mathrm{Rd}}=r_{2} W_{\mathrm{pl}, z}\left(1-\rho_{y}\right) f_{\mathrm{yd}},
\end{aligned}
$$

with $r_{1}, r_{2}$ reduction factors for axial force, $W_{\mathrm{pl}, i}$ the plastic section modulus, and $\rho_{i}(i=y, z)$ the reduction factor for uni-axial shear, obtained by considering only the contribution of $V_{i \text {,Ed }}$ in Equation (A.5). However, for I and $\mathrm{H}$ crosssections of Class 1 and 2, the reduced bending resistance $M_{\mathrm{NV}, y, \mathrm{Rd}}$ may be taken as [EC3: Eq. 6.30]:

$$
M_{\mathrm{NV}, y, \mathrm{Rd}}=r_{1}\left(W_{\mathrm{pl}, y}-\rho_{z} \frac{A_{\mathrm{w}}^{2}}{4 t_{\mathrm{w}}}\right) f_{\mathrm{yd}},
$$

where $t_{\mathrm{w}}$ represents the web thickness and $A_{\mathrm{w}}=t_{\mathrm{w}} h_{\mathrm{w}}$ the web area. The reduction factors $r_{1}$ and $r_{2}$ for axial force depend on the type of the crosssection. For I and $\mathrm{H}$ cross-sections, they are:

$$
\begin{aligned}
& r_{1}=\frac{1-\max (n, 0.5 a)}{1-0.5 a}, \\
& r_{2}=1-\left(\frac{\max (n, a)-a}{1-a}\right)^{2},
\end{aligned}
$$

with $n=\left|N_{\mathrm{Ed}}\right| / N_{\mathrm{pl}, \mathrm{Rd}}, a=\min \left(1-2 b t_{\mathrm{f}} / A, 0.5\right), b$ the width of the crosssection, $t_{\mathrm{f}}$ the flange thickness, and $A$ the area [EC3: Eqs. 6.37 to 6.40]. For SHS sections, $r_{1}$ is used for bending about either axis. Utilization ratios for bi-axial bending of Class 1 and 2 cross-sections are

$$
\mathrm{UR}_{\mathrm{R}, \mathrm{C}, 1,2, e p l}=\left(\frac{\left|M_{y, \mathrm{Ed}}\right|}{M_{\mathrm{NV}, y, \mathrm{Rd}}}\right)^{\alpha}+\left(\frac{\left|M_{z, \mathrm{Ed}}\right|}{M_{\mathrm{NV}, z, \mathrm{Rd}}}\right)^{\beta},
$$

where $M_{\mathrm{NV}, y, \mathrm{Rd}}$ and $M_{\mathrm{NV}, z, \mathrm{Rd}}$ are given by Equations (A.8), (A.9) and (A.10), using the reduction factor $\rho_{y z}$ for bi-axial shear from Equation (A.5) instead of $\rho_{i}$. Powers $\alpha$ and $\beta$ are equal to

$$
\begin{aligned}
& \alpha=2 \\
& \beta=\max (5 n, 1)
\end{aligned}
$$

for $\mathrm{I}$ and $\mathrm{H}$ sections, and to

$$
\alpha=\beta=\frac{1.66}{1-\min \left(1.13 n^{2}, 1-\frac{1.66}{6}\right)}
$$

for SHS sections.

Finally, the capacity in torsion is evaluated as follows:

$$
\mathrm{UR}_{\mathrm{R}, \mathrm{T}, e p l}=\frac{\left|T_{\mathrm{Ed}}\right|}{T_{\mathrm{Rd}}}, \quad \text { with } T_{\mathrm{Rd}}=\frac{T_{\mathrm{el}, \mathrm{t}} f_{\mathrm{yd}}}{\sqrt{3}}
$$

for all cross-section classes, where $T_{\mathrm{el}, \mathrm{t}}$ is the section modulus for St. Venant torsion (ignoring the effect of warping). 


\section{A.2. Buckling resistance of members}

The following paragraphs describe the utilization ratios $\mathrm{UR}_{\mathrm{S}}$ for stability. Buckling of structural members is taken into account by multiplying the crosssectional resistance by an appropriate reduction factor $\chi$, corresponding to the relevant buckling mode. The reduction factors are defined as:

$$
\chi=\min \left(\frac{1}{\phi+\sqrt{\phi^{2}-\lambda^{2}}}, 1\right),
$$

where $\phi=0.5\left(1+\alpha_{\text {buck }}(\lambda-0.2)+\lambda^{2}\right), \lambda$ is the non-dimensional slenderness of the member and $\alpha_{\text {buck }}$ is an imperfection factor that takes into account geometrical and material imperfections, obtained from [EC3: Tables 6.1 to 6.4]. The nondimensional slenderness $\lambda$ is computed (separately for the different buckling modes) as follows for flexural buckling:

$$
\lambda_{i}=\sqrt{\frac{A f_{\mathrm{yd}}}{N_{\mathrm{cr}, i}}}, \quad \text { with } N_{\mathrm{cr}, i}=\frac{\pi^{2} E I_{i}}{L_{\mathrm{cr}, i}^{2}} \quad(i=y, z),
$$

as follows for torsional buckling:

$$
\lambda_{\mathrm{T}}=\sqrt{\frac{A f_{\mathrm{yd}}}{N_{\mathrm{cr}, \mathrm{T}}}}, \quad \text { with } N_{\mathrm{cr}, \mathrm{T}}=\frac{A}{I_{y}+I_{z}}\left(G I_{\mathrm{T}}+\frac{\pi^{2} E I_{\mathrm{w}}}{L_{\mathrm{cr}, \mathrm{LT}}^{2}}\right),
$$

and as follows for lateral-torsional buckling:

$$
\lambda_{\mathrm{LT}}=\sqrt{\frac{W_{y} f_{\mathrm{yd}}}{M_{\mathrm{cr}}}}, \quad \text { with } M_{\mathrm{cr}}=C_{1} \frac{\pi^{2} E I_{z}}{L_{\mathrm{cr}, \mathrm{LT}}^{2}} \sqrt{\frac{I_{\mathrm{w}}}{I_{z}}+\frac{L_{\mathrm{cr}, \mathrm{LT}}^{2} G I_{\mathrm{T}}}{\pi^{2} E I_{z}}},
$$

where $A$ and $W_{y}$ are plastic, elastic or effective section properties depending on the cross-section class. In these equations, $I_{i}$ is the moment of inertia, $I_{\mathrm{T}}$ the torsion constant, $I_{\mathrm{w}}$ the warping constant, $E$ the modulus of elasticity, $G$ the shear modulus, and $L_{\mathrm{cr}, y}, L_{\mathrm{cr}, z}, L_{\mathrm{cr}, \mathrm{LT}}$ the buckling lengths for flexural and lateral-torsional buckling. The equivalent uniform moment factor $C_{1}$ for lateral-torsional buckling is obtained from the following formula by Serna et al. [49]:

$$
C_{1}=\sqrt{\frac{35 \bar{M}_{y, \mathrm{Ed}}^{2}}{\bar{M}_{y, \mathrm{Ed}}^{2}+9 M_{y, 0.25 \mathrm{~L}}^{2}+16 M_{y, 0.5 \mathrm{~L}}^{2}+9 M_{y, 0.75 \mathrm{~L}}^{2}}},
$$

where $\bar{M}_{y, \text { Ed }}$ is the maximum bending moment in the member and $M_{y, 0.25 \mathrm{~L}}$, $M_{y, 0.5 \mathrm{~L}}$ and $M_{y, 0.75 \mathrm{~L}}$ the bending moment at points $L / 4, L / 2$ and $3 L / 4$ in a member with length $L$. A member is considered insensitive (not susceptible) to torsional deformations when either $I_{\mathrm{T}} \geq I_{y}$ (e.g. for SHS sections) or

$$
\sqrt{\frac{W_{y} f_{\mathrm{yd}}}{M_{\mathrm{cr}, 0}}} \leq 0.2 C_{1} \sqrt[4]{\left(1-\frac{N_{\mathrm{Ed}}}{N_{\mathrm{cr}, z}}\right)\left(1-\frac{N_{\mathrm{Ed}}}{N_{\mathrm{cr}, \mathrm{T}}}\right)}
$$


(with $M_{\mathrm{cr}, 0}$ obtained by setting $C_{1}$ equal to 1 in Equation (A.21)), in which case $\chi_{\mathrm{LT}}$ may be taken as 1 [ANB: Annex A \& Annex D].

The utilization ratios for buckling are considered next. The design yield strength is:

$$
f_{\mathrm{yd}}=\frac{f_{\mathrm{yk}}}{\gamma_{\mathrm{M} 1}}
$$

again with $\gamma_{\mathrm{M} 1}=1$. The buckling checks relate to compression forces only, obtained as

$$
N_{\mathrm{c}, \mathrm{Ed}}=\min \left(N_{\mathrm{Ed}}, 0\right),
$$

where the minus sign is preserved for consistency of convention. Accordingly, the utilization ratio for torsional buckling is:

$$
\mathrm{UR}_{\mathrm{S}, \mathrm{T}, e p l}=-\frac{N_{\mathrm{c}, \mathrm{Ed}}}{\chi_{\mathrm{T}} N_{\mathrm{c}, \mathrm{Rd}}},
$$

where $N_{\mathrm{c}, \mathrm{Rd}}$ is the same as before (since $\gamma_{\mathrm{M} 0}=\gamma_{\mathrm{M} 1}$ ). The utilization ratio for lateral-torsional buckling is:

$$
\mathrm{UR}_{\mathrm{S}, \mathrm{LT}, e p l}=\frac{\left|M_{y, \mathrm{Ed}}\right|}{\chi_{\mathrm{LT}} M_{y, \mathrm{Rd}}},
$$

with $M_{y, \mathrm{Rd}}=W_{i} f_{\mathrm{yd}}$ and $W_{y}=W_{\mathrm{pl}, y}, W_{\mathrm{el}, y}$ or $W_{\mathrm{eff}, y}$ for Class $1 \& 2$, Class 3 and Class 4 cross-sections, respectively. The combination of flexural and lateraltorsional buckling gives the following utilization ratios for in-plane and out-ofplane buckling:

$$
\begin{aligned}
\mathrm{UR}_{\mathrm{S}, \mathrm{C}, y, e p l} & =-\frac{N_{\mathrm{c}, \mathrm{Ed}}}{\chi_{y} N_{\mathrm{c}, \mathrm{Rd}}}+\frac{k_{y y}}{\chi_{\mathrm{LT}}} \frac{\bar{M}_{y, \mathrm{Ed}}}{M_{y, \mathrm{Rd}}}+k_{y z} \frac{\bar{M}_{z, \mathrm{Ed}}}{M_{z, \mathrm{Rd}}}, \\
\mathrm{UR}_{\mathrm{S}, \mathrm{C}, z, e p l} & =-\frac{N_{\mathrm{c}, \mathrm{Ed}}}{\chi_{z} N_{\mathrm{c}, \mathrm{Rd}}}+\frac{k_{z y}}{\chi_{\mathrm{LT}}} \frac{\bar{M}_{y, \mathrm{Ed}}}{M_{y, \mathrm{Rd}}}+k_{z z} \frac{\bar{M}_{z, \mathrm{Ed}}}{M_{z, \mathrm{Rd}}} .
\end{aligned}
$$

Herein, the terms $\bar{M}_{y, \text { Ed }}$ and $\bar{M}_{z \text {,Ed }}$ denote the maximum bending moment (in absolute value) in all interpolation points along the member. The factors $k_{y y}$, $k_{y z}, k_{z y}$ and $k_{z z}$ are interaction factors that account for the effect of plasticity on the distribution of stresses in the member. The Eurocode gives two options for calculating the interaction factors, following either Annex A or Annex B. The approaches mainly differ in terms of transparency: Annex A aims to give a one-to-one representation of reality, whereas Annex B is based on the concept of global factors, aiming to derive user-friendly formulae [50]. The latter procedure is adopted in this study, because it can more easily be modified for gradientbased optimization. Accordingly, the interaction factors for Class 1 and 2 crosssections are computed as: 


$$
\begin{aligned}
k_{y y} & =C_{\mathrm{my}}\left(1-\left(\bar{\lambda}_{y}-0.2\right) \frac{N_{\mathrm{c}, \mathrm{Ed}}}{\chi_{y} N_{\mathrm{c}, \mathrm{Rd}}}\right) \\
k_{y z} & =0.6 k_{z z} \\
k_{z y} & =0.6 k_{y y} \\
k_{z z} & =C_{\mathrm{mz}}\left(1-\xi \frac{N_{\mathrm{c}, \mathrm{Ed}}}{\chi_{z} N_{\mathrm{c}, \mathrm{Rd}}}\right)
\end{aligned}
$$

with $\xi=\left(2 \bar{\lambda}_{z}-0.6\right)$ for I and H sections and $\xi=\left(\bar{\lambda}_{z}-0.2\right)$ for SHS sections, and $\vec{\lambda}_{i}=\min \left(\lambda_{i}, 1\right)$. For Class 3 and 4 cross-sections, the interaction factors are:

$$
\begin{aligned}
k_{y y} & =C_{\mathrm{my}}\left(1-0.6 \bar{\lambda}_{y} \frac{N_{\mathrm{c}, \mathrm{Ed}}}{\chi_{y} N_{\mathrm{c}, \mathrm{Rd}}}\right) \\
k_{y z} & =k_{z z} \\
k_{z y} & =0.8 k_{y y} \\
k_{z z} & =C_{\mathrm{mz}}\left(1-0.6 \bar{\lambda}_{z} \frac{N_{\mathrm{c}, \mathrm{Ed}}}{\chi_{z} N_{\mathrm{c}, \mathrm{Rd}}}\right) .
\end{aligned}
$$

The expressions above correspond to those in [EC3: Tables B.1 \& B.2], amended for the minus sign in the conventions. For members susceptible to torsional deformations, the expression for $k_{z y}$ is replaced by:

$$
k_{z y}=1+\frac{0.10 \min \left(\lambda_{z}, 1\right)}{\left(C_{\mathrm{mLT}}-0.25\right)} \frac{N_{\mathrm{c}, \mathrm{Ed}}}{\chi_{z} N_{\mathrm{Rd}}}
$$

for Class 1 and 2 cross-sections, and

$$
k_{z y}=1+\frac{0.05 \min \left(\lambda_{z}, 1\right)}{\left(C_{\mathrm{mLT}}-0.25\right)} \frac{N_{\mathrm{c}, \mathrm{Ed}}}{\chi_{z} N_{\mathrm{Rd}}}
$$

for Class 3 and 4 cross-sections. The equivalent uniform moment factors $C_{\mathrm{my}}$, $C_{\mathrm{mz}}$ and $C_{\mathrm{mLT}}$ depend on the bending moment distribution between supported points and are obtained from Table A.6, after [EC3: Table B.3].

\section{B. Member groups and section catalog}

The structural member groups are shown in Figures B.23 and B.24. There are 15 member groups for the braced frame and 13 for the Market Hall (based on the member groups in the original design). The cross-section in each member group is controlled by a single design variable. The available profiles are listed in Tables B.7 and B.8, with profile properties taken from the ArcelorMittal section catalog (according to [EN 10365: 2017]) and from the product standard [EN 10210-2: 2006] (for the SHS sections). For the braced frame, the profiles of the floor and roof beams (G1 and G2) are limited to IPE sections, those of 
Table A.6: Equivalent uniform moment factors, based on the bending moment diagram between the relevant braced points (bending about the $y$-axis and points braced in the $z$-direction for $C_{\mathrm{my}}$, bending about the $z$-axis and points braced in the $y$-direction for $C_{\mathrm{mz}}$, and bending about the $y$-axis and points braced in the $y$-direction for $C_{\mathrm{mLT}}$ ). The value of $M_{s}$ represents the bending moment at the center of the member and that of $M_{h}$ the largest bending moment value (in absolute value) at the member ends.

\begin{tabular}{|c|c|c|c|c|}
\hline Situation & Moment diagram & \multicolumn{2}{|c|}{ Range } & $C_{\mathrm{my}}, C_{\mathrm{mz}}$ and $C_{\mathrm{mLT}}$ \\
\hline \multirow{2}{*}{$\left|M_{s}\right| \leq\left|M_{h}\right|$} & $M_{1}$ & $0 \leq \alpha_{s} \leq 1$ & $-1 \leq \psi \leq 1$ & $0.2+0.8 \alpha_{s} \geq 0.4$ \\
\hline & $\alpha_{s}=M_{s} / M_{h}$ & $-1 \leq \alpha_{s}<0$ & $\begin{array}{r}0 \leq \psi \leq 1 \\
-1 \leq \psi<0\end{array}$ & $\begin{array}{c}0.1-0.8 \alpha_{s} \geq 0.4 \\
0.1(1-\psi)-0.8 \alpha_{s} \geq 0.4\end{array}$ \\
\hline \multirow{3}{*}{$\left|M_{s}\right|>\left|M_{h}\right|$} & & $0 \leq \alpha_{h} \leq 1$ & $-1 \leq \psi \leq 1$ & $0.95+0.05 \alpha_{h}$ \\
\hline & $M_{h} M^{M_{s}} \psi \Psi M_{h}$ & $-1<\alpha_{h}<0$ & $0 \leq \psi \leq 1$ & $0.95+0.05 \alpha_{h}$ \\
\hline & $\alpha_{h}=M_{h} / M_{s}$ & & $-1 \leq \psi<0$ & $0.95+0.05 \alpha_{h}(1+2 \psi)$ \\
\hline
\end{tabular}

the column members (G3 to G14) to HEA sections, and that of the wind braces (G15) to SHS sections. For the Market Hall, the profiles of the tie rods (G1) are limited to R sections, the profiles for the main frame (G2 to G8) to HE sections, and the other profiles (G9 to G13) to SHS sections.

\section{Section properties}

The conventions and dimensions of the cross-sections used in this paper are shown in figure C.25. The expressions for the shear area $A_{\mathrm{v}, i}(i=y, z)$ and the section modulus for St. Venant torsion $T_{\text {el,t }}$ can be found below. The other section properties can be found in the section catalog or in the corresponding product standard. For SHS sections, the outer radius $r_{\mathrm{o}}$ is calculated as 1.5 times the inner radius $r_{\mathrm{i}}$, which in turn is equal to the wall thickness $t$. The 'strong' axis always refers to the $y-y$ axis, and the 'weak' axis refers to the $\mathrm{z}-\mathrm{z}$ axis.

\section{C.1. Shear area}

The shear area for HE sections with a load acting parallel to the web is [EC3: $\S 6.2 .6(3)]$ :

$$
A_{\mathrm{v}, z}=A-2 b t_{\mathrm{f}}+\left(t_{\mathrm{w}}+2 r\right) t_{\mathrm{f}} \geq \eta t_{\mathrm{w}} h_{\mathrm{w}}
$$

with $\eta$ conservatively taken equal to 1 . For loads parallel to the flanges, no expression is given in EC3. Diamonds uses the following formula:

$$
A_{\mathrm{v}, y}=2 b t_{\mathrm{f}}+t_{\mathrm{w}}+r t_{\mathrm{w}} .
$$

For SHS sections, the shear area is:

$$
A_{\mathrm{v}, y}=A_{\mathrm{v}, z}=A \frac{h}{b+h}
$$


Table B.7: Profile names and indices for the braced frame problem.

\begin{tabular}{|c|c|c|c|c|c|c|c|c|c|}
\hline $\mathrm{Idx}$. & Name & Idx. & Name & Idx. & Name & Idx. & Name & Idx. & Name \\
\hline 1 & IPE 80 & 25 & HE $120 \mathrm{~A}$ & 49 & SHS $40 \times 3,2$ & 73 & SHS $90 \times 4$ & 97 & SHS $150 \times 14,2$ \\
\hline 2 & IPE 100 & 26 & $\mathrm{HE} 140 \mathrm{~A}$ & 50 & SHS $40 \times 4$ & 74 & SHS $90 \times 5$ & 98 & SHS $150 \times 16$ \\
\hline 3 & IPE 120 & 27 & $\mathrm{HE} 160 \mathrm{~A}$ & 51 & SHS $40 \times 5$ & 75 & SHS $90 \times 6,3$ & 99 & SHS $160 \times 5$ \\
\hline 4 & IPE 140 & 28 & $\mathrm{HE} 180 \mathrm{~A}$ & 52 & SHS $50 \times 2,6$ & 76 & SHS $90 \times 8$ & 100 & SHS $160 \times 6,3$ \\
\hline 5 & IPE 160 & 29 & $\mathrm{HE} 200 \mathrm{~A}$ & 53 & SHS $50 \times 3,2$ & 77 & SHS $100 \times 4$ & 101 & SHS $160 \times 8$ \\
\hline 6 & IPE 180 & 30 & HE $220 \mathrm{~A}$ & 54 & SHS $50 \times 4$ & 78 & SHS $100 \times 5$ & 102 & SHS $160 \times 10$ \\
\hline 7 & IPE 200 & 31 & HE $240 \mathrm{~A}$ & 55 & SHS $50 \times 5$ & 79 & SHS $100 \times 6,3$ & 103 & SHS $160 \times 12,5$ \\
\hline 8 & IPE 220 & 32 & HE $260 \mathrm{~A}$ & 56 & SHS $50 \times 6,3$ & 80 & SHS $100 \times 8$ & 104 & SHS $160 \times 14,2$ \\
\hline 9 & IPE 240 & 33 & $\mathrm{HE} 280 \mathrm{~A}$ & 57 & SHS $60 \times 2,6$ & 81 & SHS $100 \times 10$ & 105 & SHS $160 \times 16$ \\
\hline 10 & IPE 270 & 34 & HE $300 \mathrm{~A}$ & 58 & SHS $60 \times 3,2$ & 82 & SHS $120 \times 5$ & 106 & SHS $180 \times 5$ \\
\hline 11 & IPE 300 & 35 & HE $320 \mathrm{~A}$ & 59 & SHS $60 \times 4$ & 83 & SHS $120 \times 6,3$ & 107 & SHS $180 \times 6,3$ \\
\hline 12 & IPE 330 & 36 & $\mathrm{HE} 340 \mathrm{~A}$ & 60 & SHS $60 \times 5$ & 84 & SHS $120 \times 8$ & 108 & SHS $180 \times 8$ \\
\hline 13 & IPE 360 & 37 & HE $360 \mathrm{~A}$ & 61 & SHS $60 \times 6,3$ & 85 & SHS $120 \times 10$ & 109 & SHS $180 \times 10$ \\
\hline 14 & IPE 400 & 38 & $\mathrm{HE} 400 \mathrm{~A}$ & 62 & SHS $60 \times 8$ & 86 & SHS $120 \times 12,5$ & 110 & SHS $180 \times 12,5$ \\
\hline 15 & IPE 450 & 39 & $\mathrm{HE} 450 \mathrm{~A}$ & 63 & SHS $70 \times 3,2$ & 87 & SHS $140 \times 5$ & 111 & SHS $180 \times 14,2$ \\
\hline 16 & IPE 500 & 40 & HE $500 \mathrm{~A}$ & 64 & SHS $70 \times 4$ & 88 & SHS $140 \times 6,3$ & 112 & SHS $180 \times 16$ \\
\hline 17 & IPE 550 & 41 & HE $550 \mathrm{~A}$ & 65 & SHS $70 \times 5$ & 89 & SHS $140 \times 8$ & 113 & SHS $200 \times 5$ \\
\hline 18 & IPE 600 & 42 & $\mathrm{HE} 600 \mathrm{~A}$ & 66 & SHS $70 \times 6,3$ & 90 & SHS $140 \times 10$ & 114 & SHS $200 \times 6,3$ \\
\hline 19 & IPE $750 \times 134$ & 43 & $\mathrm{HE} 650 \mathrm{~A}$ & 67 & SHS $70 \times 8$ & 91 & SHS $140 \times 12,5$ & 115 & SHS $200 \times 8$ \\
\hline 20 & IPE $750 \times 147$ & 44 & $\mathrm{HE} 700 \mathrm{~A}$ & 68 & SHS $80 \times 3,2$ & 92 & SHS $150 \times 5$ & 116 & SHS $200 \times 10$ \\
\hline 21 & IPE $750 \times 173$ & 45 & HE $800 \mathrm{~A}$ & 69 & SHS $80 \times 4$ & 93 & SHS $150 \times 6,3$ & 117 & SHS $200 \times 12,5$ \\
\hline 22 & IPE $750 \times 196$ & 46 & HE $900 \mathrm{~A}$ & 70 & SHS $80 \times 5$ & 94 & SHS $150 \times 8$ & 118 & SHS $200 \times 14,2$ \\
\hline 23 & IPE $750 \times 220$ & 47 & HE 1000 A & 71 & SHS $80 \times 6,3$ & 95 & SHS $150 \times 10$ & 119 & SHS $200 \times 16$ \\
\hline 24 & HE $100 \mathrm{~A}$ & 48 & SHS $40 \times 2,6$ & 72 & SHS $80 \times 8$ & 96 & SHS $150 \times 12,5$ & & \\
\hline
\end{tabular}

\section{C.2. Section modulus for St. Venant torsion}

The section modulus $T_{\mathrm{el}, \mathrm{t}}$ for St. Venant torsion is computed as follows for HE sections:

$$
T_{\mathrm{el}, \mathrm{t}}=1.3 \frac{\left(2 b t_{\mathrm{f}}^{3}\right)+\left(h-2 t_{\mathrm{f}}\right) t_{\mathrm{w}}^{3}}{3 a_{\mathrm{m}}},
$$

with $a_{\mathrm{m}}=\max \left(t_{\mathrm{f}}, t_{\mathrm{w}}\right)$, and as follows for SHS sections:

$$
T_{\mathrm{el}, \mathrm{t}}=2(h-t)^{2} t
$$

\section{Load cases}

The following figures give an overview of load cases considered for the braced frame (Figures D.26 and D.27) and the Market Hall (Figure D.28).

\section{References}

[1] F. Pomponi, A. Moncaster, Reducing embodied carbon in the built environment: A research agenda, in: International Conference on Sustainable Ecological Engineering Design for Society, Leeds, UK, 2016. 
Table B.8: Profile names and indices for the Market Hall problem.

\begin{tabular}{|c|c|c|c|c|c|c|c|c|c|c|c|}
\hline $\operatorname{Idx}$. & Name & Idx. & Name & Idx. & Name & $\operatorname{Idx}$. & Name & Idx. & Name & Idx. & Name \\
\hline 1 & R 30 & 26 & R 55 & 51 & SHS $180 \times 16$ & 76 & SHS $260 \times 16$ & 101 & HE $140 \mathrm{M}$ & 126 & HE $320 \mathrm{~A}$ \\
\hline 2 & R 31 & 27 & R 55.8 & 52 & SHS $200 \times 5$ & 77 & SHS $300 \times 6.3$ & 102 & HE $160 \mathrm{~A}$ & 127 & HE $320 \mathrm{~B}$ \\
\hline 3 & R 32 & 28 & R 56 & 53 & SHS $200 \times 6.3$ & 78 & SHS $300 \times 8$ & 103 & HE $160 \mathrm{~B}$ & 128 & HE $320 \mathrm{M}$ \\
\hline 4 & R 34 & 29 & R 57 & 54 & SHS $200 \times 8$ & 79 & SHS $300 \times 10$ & 104 & HE $160 \mathrm{M}$ & 129 & HE $340 \mathrm{~A}$ \\
\hline 5 & R 34.4 & 30 & R 58 & 55 & SHS $200 \times 10$ & 80 & SHS $300 \times 12.5$ & 105 & HE $180 \mathrm{~A}$ & 130 & HE $340 \mathrm{~B}$ \\
\hline 6 & R 35 & 31 & R 59 & 56 & SHS $200 \times 12.5$ & 81 & SHS $300 \times 14.2$ & 106 & HE $180 \mathrm{~B}$ & 131 & HE $340 \mathrm{M}$ \\
\hline 7 & R 35.7 & 32 & $\mathrm{R} 60$ & 57 & SHS $200 \times 14.2$ & 82 & SHS $300 \times 16$ & 107 & HE $180 \mathrm{M}$ & 132 & HE $360 \mathrm{~A}$ \\
\hline 8 & R 36 & 33 & $\mathrm{R} 62$ & 58 & SHS $200 \times 16$ & 83 & SHS $350 \times 8$ & 108 & $\mathrm{HE} 200 \mathrm{~A}$ & 133 & HE $360 \mathrm{~B}$ \\
\hline 9 & $\mathrm{R} 37$ & 34 & R 63 & 59 & SHS $220 \times 6.3$ & 84 & SHS $350 \times 10$ & 109 & HE $200 \mathrm{~B}$ & 134 & HE $360 \mathrm{M}$ \\
\hline 10 & R 38 & 35 & R 65 & 60 & SHS $220 \times 8$ & 85 & SHS $350 \times 12.5$ & 110 & HE $200 \mathrm{M}$ & 135 & HE $400 \mathrm{~A}$ \\
\hline 11 & R 39 & 36 & R 70 & 61 & SHS $220 \times 10$ & 86 & SHS $350 \times 14.2$ & 111 & HE $220 \mathrm{~A}$ & 136 & HE $400 \mathrm{~B}$ \\
\hline 12 & R 39.2 & 37 & R 75 & 62 & SHS $220 \times 12.5$ & 87 & SHS $350 \times 16$ & 112 & HE $220 \mathrm{~B}$ & 137 & HE $400 \mathrm{M}$ \\
\hline 13 & R 40 & 38 & $\mathrm{R} 80$ & 63 & SHS $220 \times 14.2$ & 88 & SHS $400 \times 10$ & 113 & HE $220 \mathrm{M}$ & 138 & $\mathrm{HE} 450 \mathrm{~A}$ \\
\hline 14 & R 42 & 39 & $\mathrm{R} 85$ & 64 & SHS $220 \times 16$ & 89 & SHS $400 \times 12.5$ & 114 & HE $240 \mathrm{~A}$ & 139 & $\mathrm{HE} 450 \mathrm{~B}$ \\
\hline 15 & $\mathrm{R} 44$ & 40 & $\mathrm{R} 90$ & 65 & SHS $250 \times 6.3$ & 90 & SHS $400 \times 14.2$ & 115 & $\mathrm{HE} 240 \mathrm{~B}$ & 140 & HE $450 \mathrm{M}$ \\
\hline 16 & $\mathrm{R} 45$ & 41 & R 95 & 66 & SHS $250 \times 8$ & 91 & SHS $400 \times 16$ & 116 & HE $240 \mathrm{M}$ & 141 & $\mathrm{HE} 500 \mathrm{~A}$ \\
\hline 17 & $\mathrm{R} 46$ & 42 & R 100 & 67 & SHS $250 \times 10$ & 92 & SHS $400 \times 20$ & 117 & HE $260 \mathrm{~A}$ & 142 & HE $500 \mathrm{~B}$ \\
\hline 18 & $\mathrm{R} 47$ & 43 & R 105 & 68 & SHS $250 \times 12.5$ & 93 & HE $100 \mathrm{~A}$ & 118 & HE $260 \mathrm{~B}$ & 143 & HE $500 \mathrm{M}$ \\
\hline 19 & $\mathrm{R} 48$ & 44 & R 110 & 69 & SHS $250 \times 14.2$ & 94 & HE $100 \mathrm{~B}$ & 119 & HE $260 \mathrm{M}$ & 144 & $\mathrm{HE} 550 \mathrm{~A}$ \\
\hline 20 & R 49.2 & 45 & SHS $180 \times 5$ & 70 & SHS $250 \times 16$ & 95 & HE $100 \mathrm{M}$ & 120 & $\mathrm{HE} 280 \mathrm{~A}$ & 145 & HE $550 \mathrm{~B}$ \\
\hline 21 & R 50 & 46 & SHS $180 \times 6.3$ & 71 & SHS $260 \times 6.3$ & 96 & $\mathrm{HE} 120 \mathrm{~A}$ & 121 & HE $280 \mathrm{~B}$ & 146 & HE $550 \mathrm{M}$ \\
\hline 22 & R 51 & 47 & SHS $180 \times 8$ & 72 & SHS $260 \times 8$ & 97 & HE $120 \mathrm{~B}$ & 122 & HE $280 \mathrm{M}$ & 147 & HE $600 \mathrm{~A}$ \\
\hline 23 & R 52 & 48 & SHS $180 \times 10$ & 73 & SHS $260 \times 10$ & 98 & $\mathrm{HE} 120 \mathrm{M}$ & 123 & HE $300 \mathrm{~A}$ & 148 & HE $600 \mathrm{~B}$ \\
\hline 24 & R 53 & 49 & SHS $180 \times 12.5$ & 74 & SHS $260 \times 12.5$ & 99 & HE $140 \mathrm{~A}$ & 124 & HE 300 B & 149 & HE $600 \mathrm{M}$ \\
\hline 25 & R 54 & 50 & SHS $180 \times 14.2$ & 75 & SHS $260 \times 14.2$ & 100 & $\mathrm{HE} 140 \mathrm{~B}$ & 125 & HE $300 \mathrm{M}$ & & \\
\hline
\end{tabular}


Table B.9: Member sections for the braced frame.

\begin{tabular}{lll}
\hline $\begin{array}{l}\text { Member } \\
\text { group }\end{array}$ & Genetic algorithm & Hybrid algorithm \\
\hline G1 & IPE 300 & IPE 270 \\
G2 & IPE 270 & IPE 220 \\
G3 & HE 260 A & HE 260 A \\
G4 & HE 240 A & HE 220 A \\
G5 & HE 200 A & HE 200 A \\
G6 & HE 200 A & HE 220 A \\
G7 & HE 180 A & HE 160 A \\
G8 & HE 120 A & HE 120 A \\
G9 & HE 240 A & HE 240 A \\
G10 & HE 220 A & HE 220 A \\
G11 & HE 200 A & HE 200 A \\
G12 & HE 180 A & HE 180 A \\
G13 & HE 160 A & HE 160 A \\
G14 & HE 140 A & HE 140 A \\
G15 & SHS $120 \times 5$ & SHS $120 \times 5$ \\
\hline Weight & 8.4 tons & 7.6 tons \\
\hline
\end{tabular}

Table B.10: Member sections for the Market Hall.

\begin{tabular}{|c|c|c|c|}
\hline $\begin{array}{l}\text { Member } \\
\text { group }\end{array}$ & $\begin{array}{l}\text { Original } \\
\text { design }\end{array}$ & $\begin{array}{l}\text { Genetic } \\
\text { algorithm }\end{array}$ & $\begin{array}{l}\text { Hybrid } \\
\text { algorithm }\end{array}$ \\
\hline G1 & R 60 & R 75 & R 65 \\
\hline $\mathrm{G} 2$ & HE $280 \mathrm{~A}$ & HE $220 \mathrm{~A}$ & HE $160 \mathrm{~B}$ \\
\hline G3 & HE $280 \mathrm{~A}$ & HE $260 \mathrm{~A}$ & HE $280 \mathrm{~A}$ \\
\hline G4 & HE $280 \mathrm{~B}$ & HE $160 \mathrm{~A}$ & HE $160 \mathrm{~A}$ \\
\hline G5 & HE 300 B & HE 180 B & HE $280 \mathrm{~A}$ \\
\hline G6 & HE 300 B & HE 300 B & HE 300 B \\
\hline G7 & HE 320 B & HE 300 B & HE 320 B \\
\hline G8 & HE 360 B & $\mathrm{HE} 280 \mathrm{M}$ & HE 360 B \\
\hline G9 & SHS $300 \times 12.5$ & SHS $300 \times 10$ & SHS $300 \times 8$ \\
\hline G10 & SHS $300 \times 16$ & SHS $250 \times 10$ & SHS $250 \times 6.3$ \\
\hline G11 & SHS $350 \times 16$ & SHS $260 \times 16$ & SHS $350 \times 14.2$ \\
\hline G12 & SHS $300 \times 16$ & SHS $300 \times 16$ & SHS $300 \times 16$ \\
\hline G13 & SHS $400 \times 20$ & SHS $350 \times 8$ & SHS $400 \times 10$ \\
\hline Weight & 226.6 tons & 191.4 tons & 184.5 tons \\
\hline
\end{tabular}

[2] R. Crawford, Life Cycle Assessment in the Built Environment, Spon Press, London, New York, 2011. 
[3] T. Ibn-Mohammed, R. Greenough, S. Taylor, L. Ozawa-Meida, A. Acquaye, Operational vs. embodied emissions in buildings - a review of current trends, Energy and Buildings 66 (2013) 232-245.

[4] M. Webster, H. Meryman, A. Slivers, T. Rodriguez-Nikl, L. Lemay, K. Simonen, Structure and carbon - how materials affect the climate, SEI Sustainability Committee, Carbon Working Group, ASCE (2012).

[5] M. Moynihan, J. Allwood, Utilization of structural steel in buildings, Proceedings of The Royal Society A 470 (2168) (2014) 20140170.

[6] F. Needham, The economics of steelwork design, The Structural Engineer 55 (9) (1977) 367-373.

[7] C. Gibbons, Economic steelwork design, The Structural Engineer 73 (15) (1995) 250-253.

[8] A. Rolvink, C. Mueller, J. Coenders, State on the art of computational tools for conceptual structural design, in: Proceedings of IASS Annual Symposia, International Association for Shell and Spatial Structures (IASS), 2014, pp. $1-8$.

[9] M. Stolpe, Truss optimization with discrete design variables: a critical review, Structural and Multidisciplinary Optimization 53 (2) (2016) 349374 .

[10] J. Nocedal, S. Wright, Numerical optimization, Springer Science \& Business Media, 2006.

[11] S. Mehrotra, On the implementation of a primal-dual interior point method, SIAM Journal on Optimization 2 (4) (1992) 575-601.

[12] K. Svanberg, The method of moving asymptotes - a new method for structural optimization, International Journal for Numerical Methods in Engineering 24 (2) (1987) 359-373.

[13] R. Haftka, Z. Gürdal, Elements of structural optimization, 3rd Edition, Kluwer Academic Publishers, Dordrecht, The Netherlands, 1992.

[14] O. Sigmund, On the usefulness of non-gradient approaches in topology optimization, Structural and Multidisciplinary Optimization 43 (5) (2011) $589-596$.

[15] J. Holland, et al., Adaptation in natural and artificial systems: an introductory analysis with applications to biology, control, and artificial intelligence, MIT Press, 1992.

[16] J. Kennedy, R. Eberhart, Particle swarm optimization, in: Proceedings of the IEEE International Conference on Neural Networks, Vol. 4, Piscataway, NJ, US, 1995, pp. 1942-1948. 
[17] S. Kirkpatrick, C. Gelatt Jr, M. Vecchi, Optimization by simulated annealing, Science 220 (4598) (1983) 671-680.

[18] O. Hasançebi, S. Azad, Adaptive dimensional search: a new metaheuristic algorithm for discrete truss sizing optimization, Computers \& Structures 154 (2015) 1-16.

[19] S. Azad, Monitored convergence curve: a new framework for metaheuristic structural optimization algorithms, Structural and Multidisciplinary Optimization 60 (2) (2019) 481-499.

[20] R. Bellman, Dynamic programming, Science 153 (3731) (1966) 34-37.

[21] F. Einabadi, M. Ebadzadeh, Multiscale gradient based swarm optimizer, in: Proceedings of the 16th CSI International Symposium on Artificial Intelligence and Signal Processing, Shiraz, Iran, 2012, pp. 049-053.

[22] V. Plevris, M. Papadrakakis, A hybrid particle swarm-gradient algorithm for global structural optimization, Computer-Aided Civil and Infrastructure Engineering 26 (1) (2011) 48-68.

[23] S. Chen, T. Mei, M. Luo, X. Yang, Identification of nonlinear system based on a new hybrid gradient-based PSO algorithm, in: Proceedings of the IEEE International Conference on Information Acquisition, Jeju City, Korea, 2007, pp. 265-268.

[24] S. Das, P. Koduru, M. Gui, M. Cochran, A. Wareing, S. Welch, B. Babin, Adding local search to particle swarm optimization, in: Proceedings of the IEEE International Conference on Evolutionary Computation, Vancouver, Canada, 2006, pp. 428-433.

[25] M. Noel, T. Jannett, Simulation of a new hybrid particle swarm optimization algorithm, in: Proceedings of the 36th Southeastern Symposium on System Theory, 2004, pp. 150-153.

[26] W. Gutkowski, J. Bauer, J. Zawidzka, An effective method for discrete structural optimization, Engineering Computations: Int J for ComputerAided Engineering 17 (4) (2000) 417-426.

[27] G. Guerlement, R. Targowski, W. Gutkowski, J. Zawidzka, J. Zawidzki, Discrete minimum weight design of steel structures using EC3 code, Structural and Multidisciplinary Optimization 22 (4) (2001) 322-327.

[28] J. Jalkanen, Tubular truss optimization using heuristic algorithms, Ph.D. thesis, Tampere University of Technology (2007).

[29] K. Mela, Resolving issues with member buckling in truss topology optimization using a mixed variable approach, Structural and Multidisciplinary Optimization 50 (6) (2014) 1037-1049. 
[30] R. Van Mellaert, G. Lombaert, M. Schevenels, Global size optimization of statically determinate trusses considering displacement, member, and joint constraints, Journal of Structural Engineering 142 (2) (2016) 04015120.

[31] R. Van Mellaert, K. Mela, T. Tiainen, M. Heinisuo, G. Lombaert, M. Schevenels, Mixed-integer linear programming approach for global discrete sizing optimization of frame structures, Structural and Multidisciplinary Optimization 57 (2) (2018) 579-593.

[32] B. D'Amico, F. Pomponi, Accuracy and reliability: A computational tool to minimise steel mass and carbon emissions at early-stage structural design, Energy and Buildings 168 (2018) 236-250.

[33] S. Rajan, Sizing, shape, and topology design optimization of trusses using genetic algorithm, Journal of Structural Engineering 121 (10) (1995) 14801487.

[34] R. Balling, R. Briggs, K. Gillman, Multiple optimum size/shape/topology designs for skeletal structures using a genetic algorithm, Journal of Structural Engineering 132 (7) (2006) 1158-1165.

[35] K. Martini, Harmony search method for multimodal size, shape, and topology optimization of structural frameworks, Journal of Structural Engineering 137 (11) (2011) 1332-1339.

[36] A. Mortazavi, V. Toğan, Simultaneous size, shape, and topology optimization of truss structures using integrated particle swarm optimizer, Structural and Multidisciplinary Optimization 54 (4) (2016) 715-736.

[37] R. Haftka, Requirements for papers focusing on new or improved global optimization algorithms, Structural and Multidisciplinary Optimization 54 (1) (2016) 1-1.

[38] W. Achtziger, On simultaneous optimization of truss geometry and topology, Structural and Multidisciplinary Optimization 33 (4-5) (2007) 285304 .

[39] S. Rajeev, C. Krishnamoorthy, Discrete optimization of structures using genetic algorithms, Journal of Structural Engineering 118 (5) (1992) 12331250 .

[40] G. Kreisselmeier, R. Steinhauser, Systematic control design by optimizing a vector performance index, in: Computer aided design of control systems, Elsevier, 1980, pp. 113-117.

[41] W. Dillen, G. Lombaert, R. Mertens, H. Van Beurden, D. Jaspaert, M. Schevenels, Optimization in a realistic structural engineering context: redesign of the Market Hall in Ghent, Engineering Structures 228 (2021) 111473 . 
[42] O. Zienkiewicz, R. Taylor, P. Nithiarasu, J. Zhu, The finite element method, Vol. 3, McGraw-hill London, 1977.

[43] KU Leuven, Stabil: A Matlab toolbox for structural mechanics, https: //bwk.kuleuven. be/bwm/stabil (March 2020).

[44] R. Mertens, Structurele optimalisatie van de Stadshal in Gent [Structural optimization of the Market Hall in Ghent], Master's thesis, Division of Architectural Engineering, KU Leuven, Leuven, Belgium (2019).

[45] BuildSoft, Stadshal, Published online, accessed 10 Oct 2019. http://www. buildsoft.eu/nl/referentieproject/ (January 2019).

[46] A. Verbart, M. Stolpe, A working-set approach for sizing optimization of frame-structures subjected to time-dependent constraints, Structural and Multidisciplinary Optimization 58 (4) (2018) 1367-1382.

[47] R. Haftka, Z. Gürdal, Elements of structural optimization, Vol. 11, Springer Science \& Business Media, 2012.

[48] S. Azad, O. Hasançebi, S. Azad, Upper bound strategy for metaheuristic based design optimization of steel frames, Advances in Engineering Software 57 (2013) 19-32.

[49] M. Serna, A. López, I. Puente, D. Yong, Equivalent uniform moment factors for lateral-torsional buckling of steel members, Journal of Constructional Steel Research 62 (6) (2006) 566-580.

[50] R. Maquoi, N. Boissonnade, J. Muzeau, J.-P. Jaspart, M. Villette, The interaction formulae for beam-columns: a new step of yet long story, in: Proceedings 2001 of the SSRC Annual Technical Session and Meeting, 2001, pp. 63-88. 

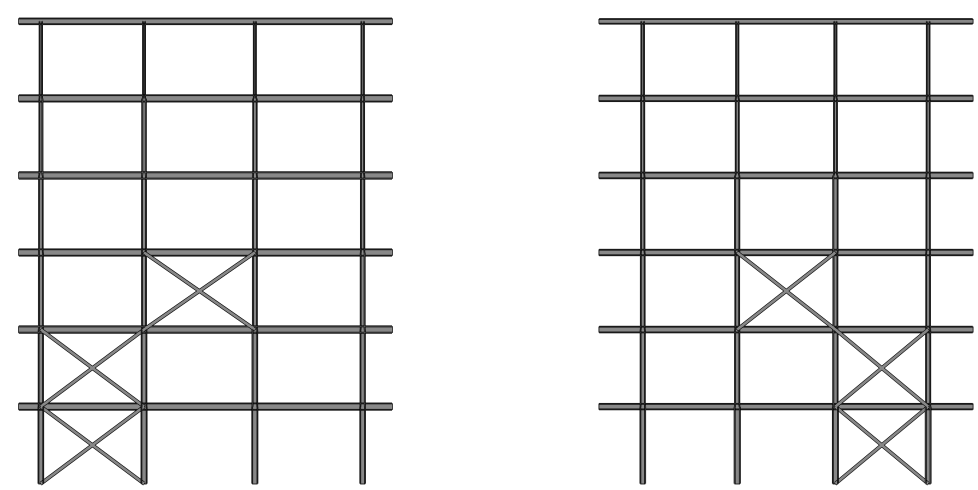

Figure 10: Genetic algorithm: geometry (8.4Figure 11: Hybrid algorithm: geometry (7.6 tons). tons).
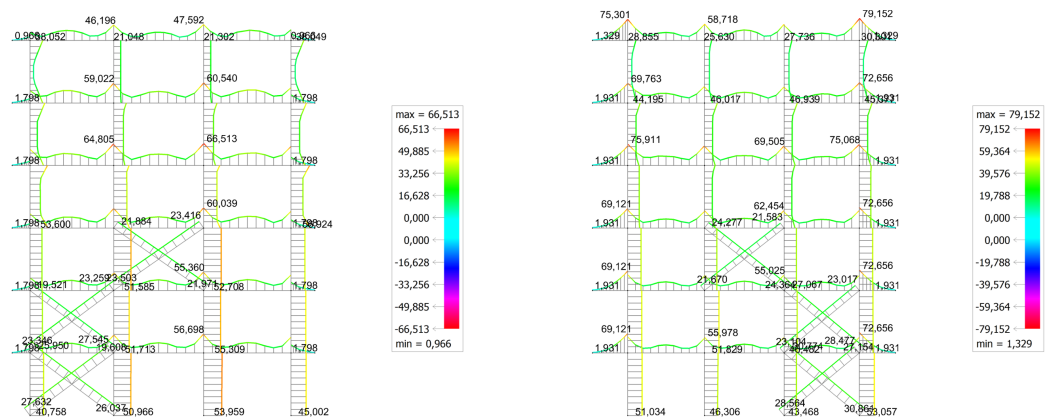

Figure 12: Genetic algorithm: $\mathrm{UR}_{\mathrm{R}}$ in Dia-Figure 13: Hybrid algorithm: $\mathrm{UR}_{\mathrm{R}}$ in Diamonds (\%). monds $(\%)$.
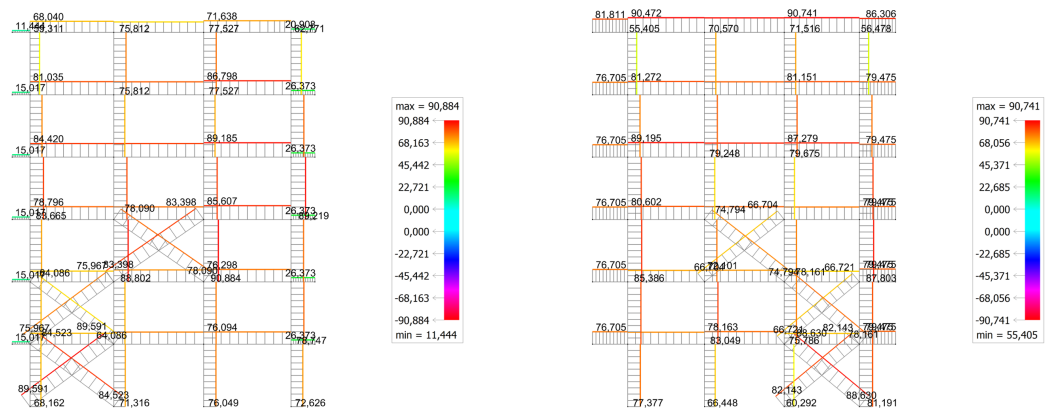

Figure 14: Genetic algorithm: $\mathrm{UR}_{\mathrm{S}}$ in Dia-Figure 15: Hybrid algorithm: $\mathrm{UR}_{\mathrm{S}}$ in Diamonds (\%). monds (\%). 


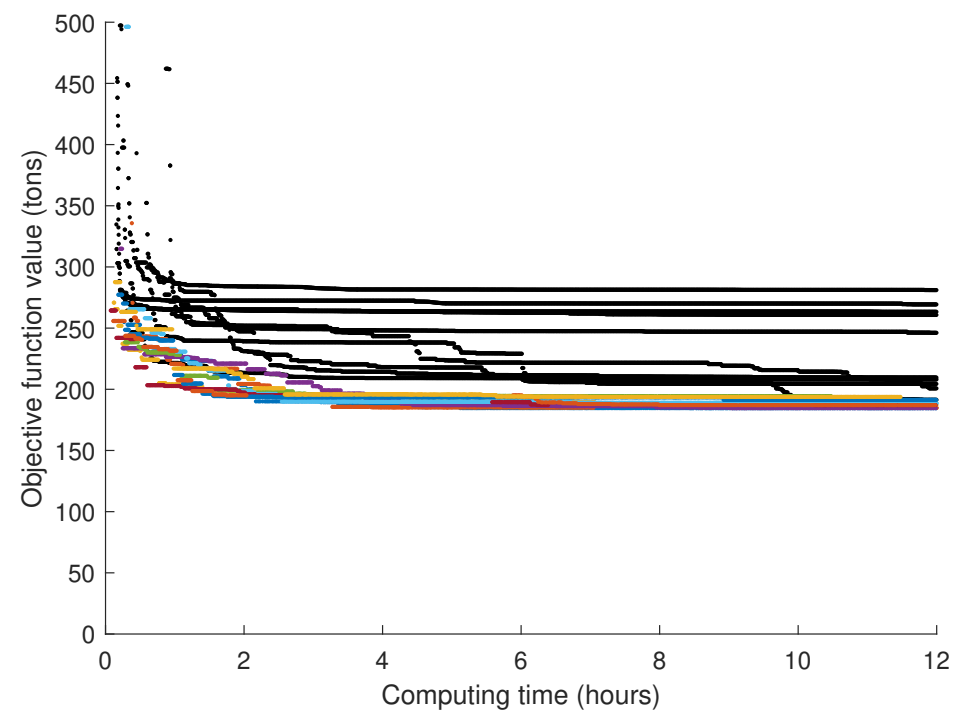

Figure 16: Convergence history for the Market Hall. 


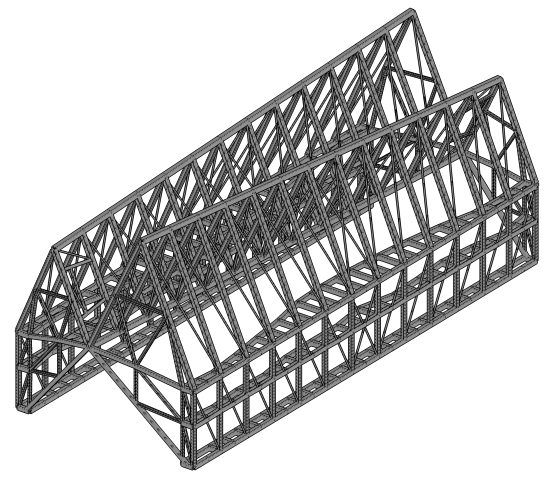

Figure 17: Genetic algorithm: geometry (191.4 tons).

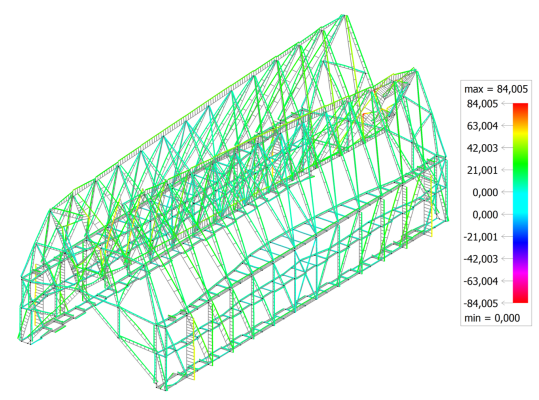

Figure 19: Genetic algorithm: $\mathrm{UR}_{\mathrm{R}}$ in Diamonds (\%).
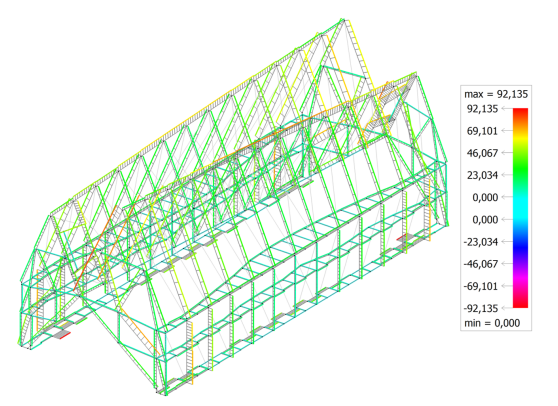

Figure 21: Genetic algorithm: $\mathrm{UR}_{\mathrm{S}}$ in Diamonds (\%).

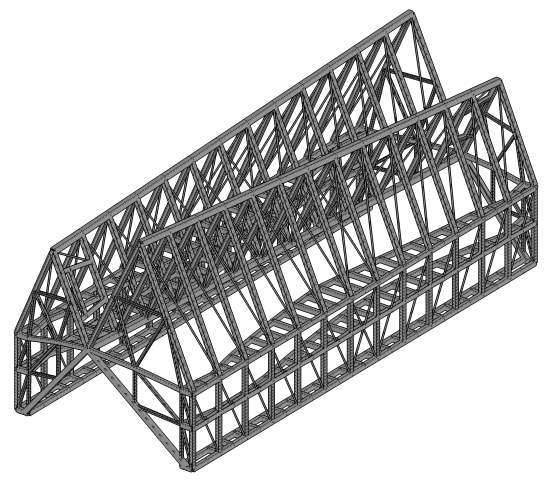

Figure 18: Hybrid algorithm: geometry (184.5 tons).

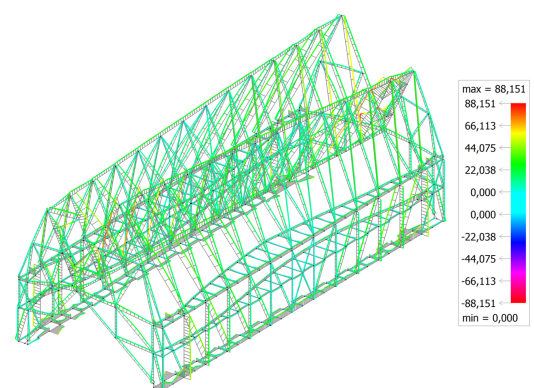

Figure 20: Hybrid algorithm: $\mathrm{UR}_{\mathrm{R}}$ in Diamonds (\%).

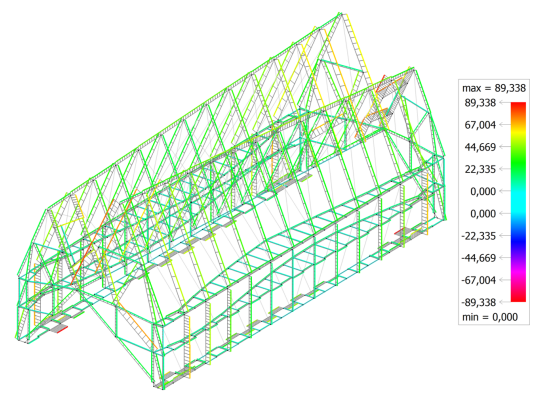

Figure 22: Hybrid algorithm: $\mathrm{UR}_{\mathrm{S}}$ in Diamonds (\%). 


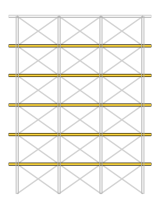

G1

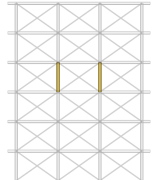

G6

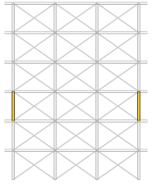

G11

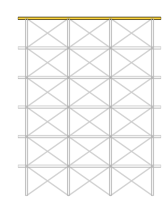

G2

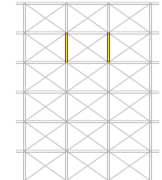

G7

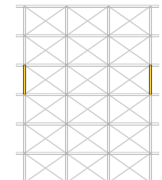

G12

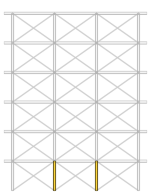

G3

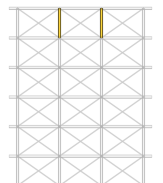

G8

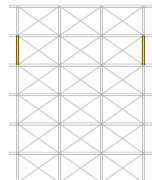

G13

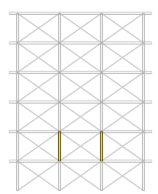

G4

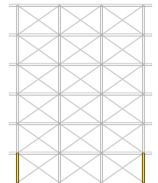

G9

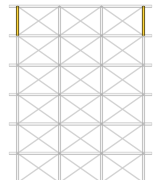

G14

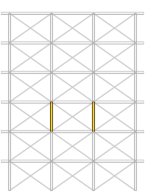

G5

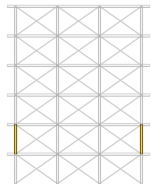

G10

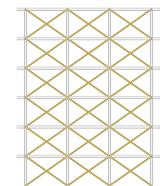

G15

Figure B.23: Structural member groups (size design variables) for the braced frame. 


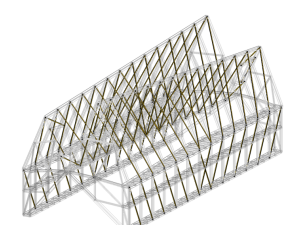

G1

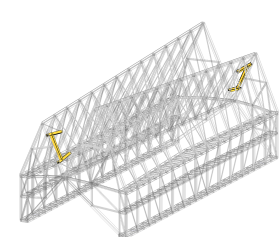

G5

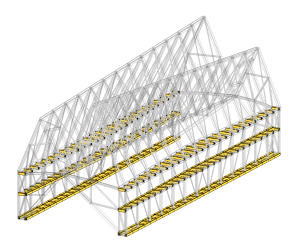

G9

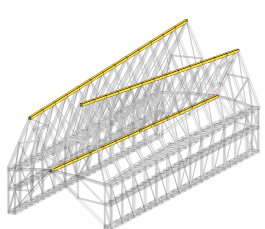

G13

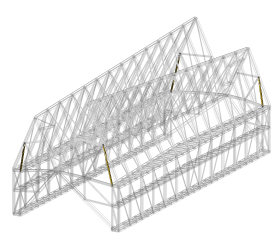

G2

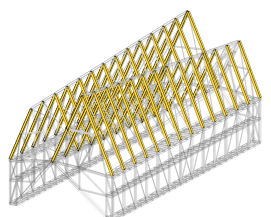

G6

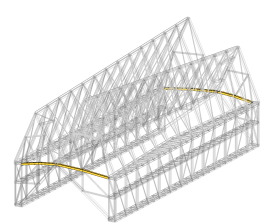

G10

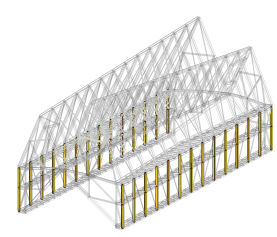

G3

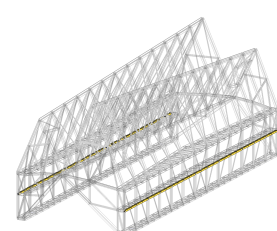

G7

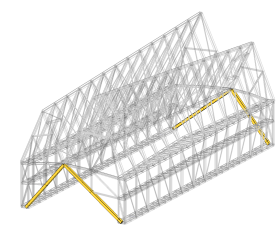

G11

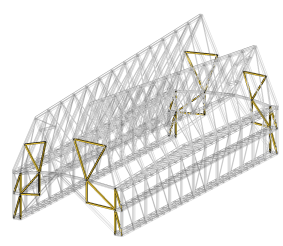

G4

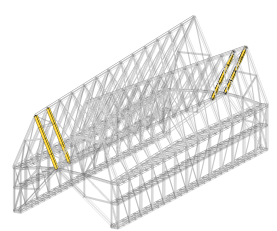

G8

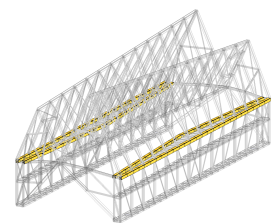

G12

Figure B.24: Structural member groups (size design variables) for the Market Hall.
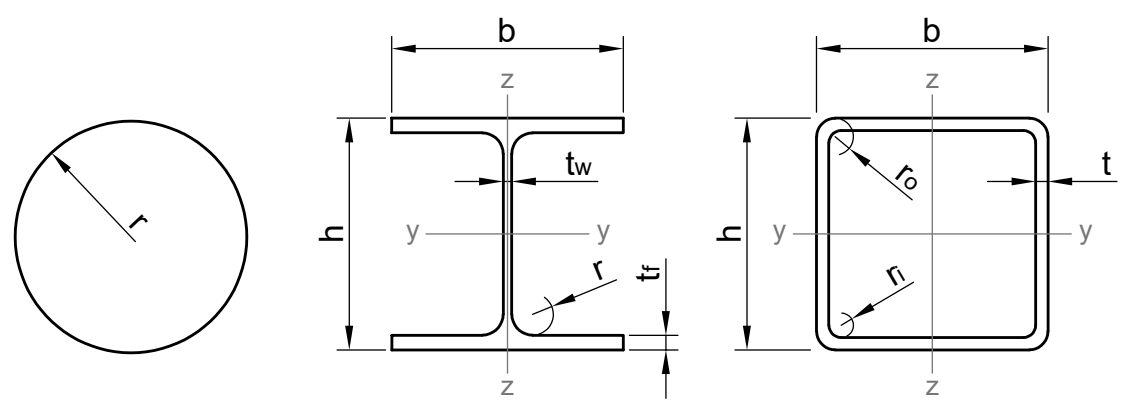

Figure C.25: Eurocode conventions for R, I/H and SHS sections. 


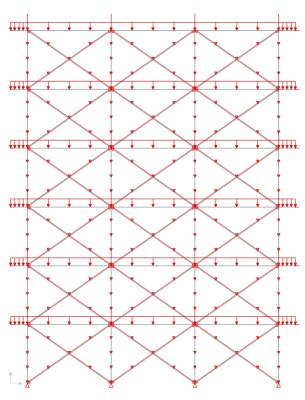

Self-weight

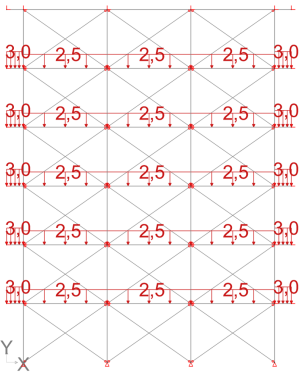

Imposed load B (1)

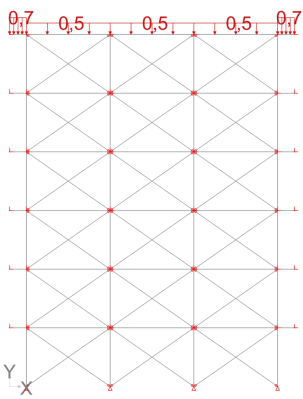

Imposed load H (1)

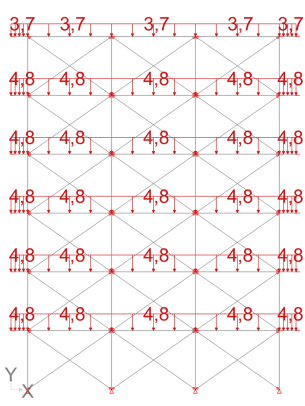

Permanent load

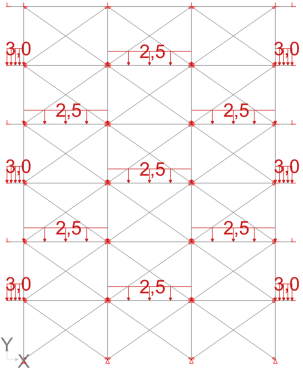

Imposed load B (2)

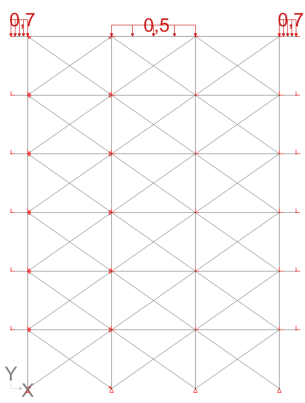

Imposed load H (2)

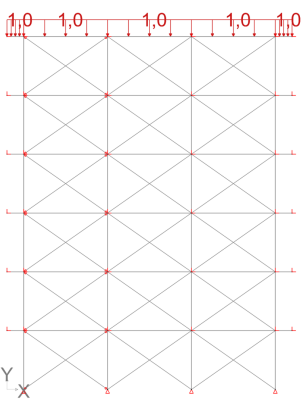

Snow load

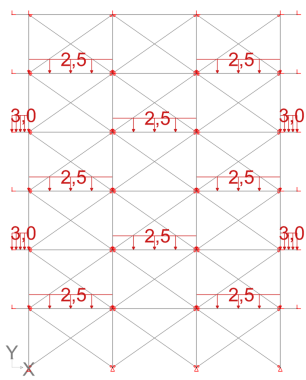

Imposed load B (3)

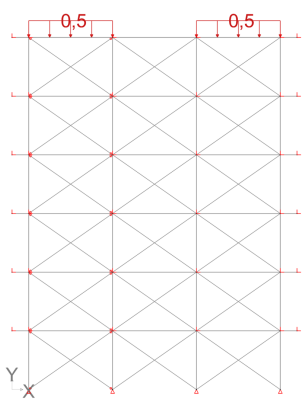

Imposed load H (3)

Figure D.26: Load cases for the braced frame. Forces are in $\mathrm{kN} / \mathrm{m}$ and distributed loads in $\mathrm{kN} / \mathrm{m}^{2}$ and need to be multiplied by the distance between subsequent frames $(6 \mathrm{~m})$. 


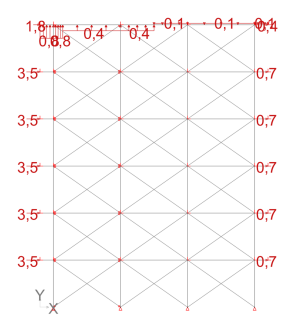

Wind (1)

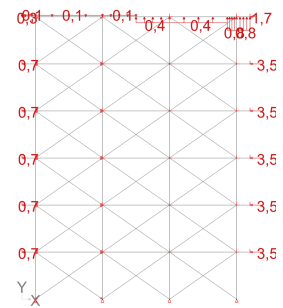

Wind (5)

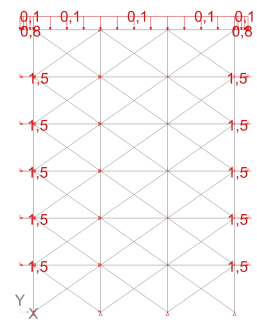

Wind (9)

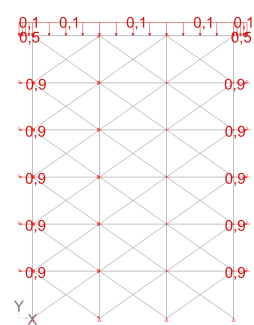

Wind (13)

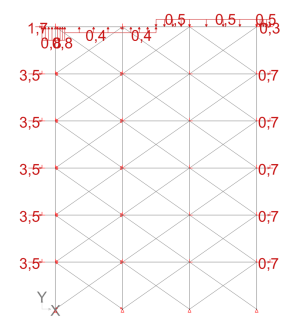

Wind (2)

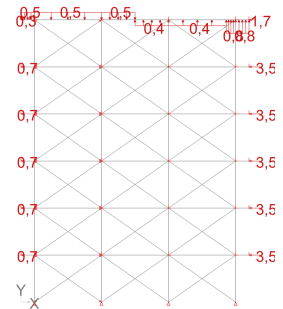

Wind (6)

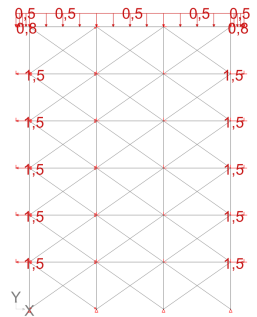

Wind (10)

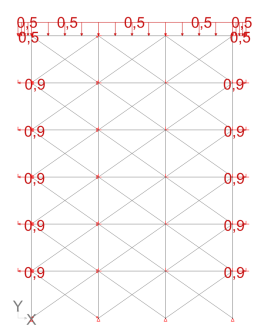

Wind (14)

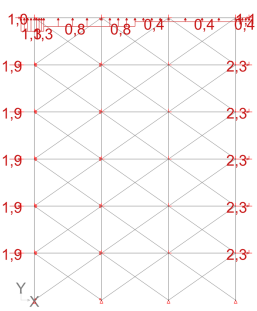

Wind (3)

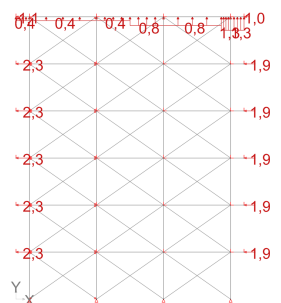

Wind (7)

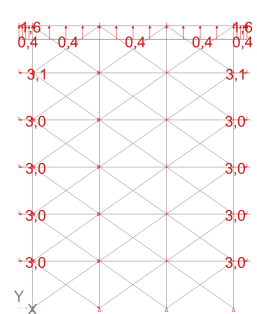

Wind (11)

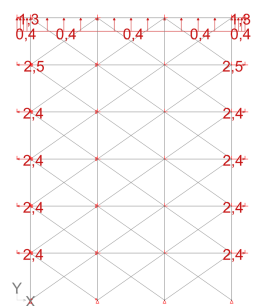

Wind (15)

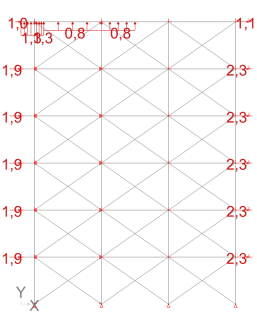

Wind (4)

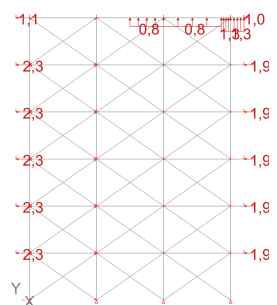

Wind (8)

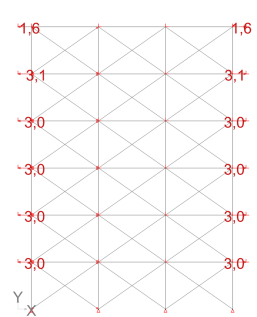

Wind (12)

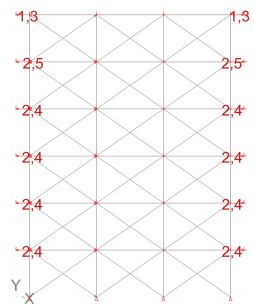

Wind (16)

Figure D.27: Load cases for the braced frame (continued). 


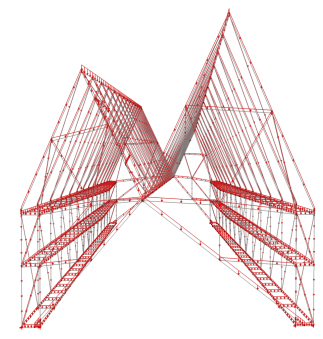

Self-weight

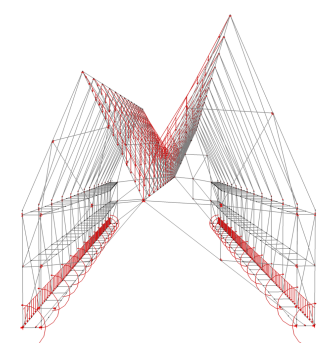

Snow load

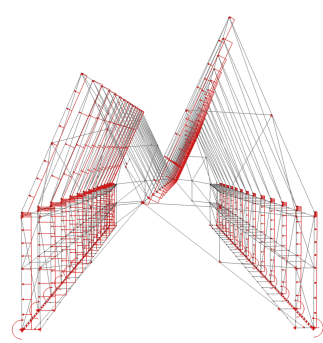

Wind (S)

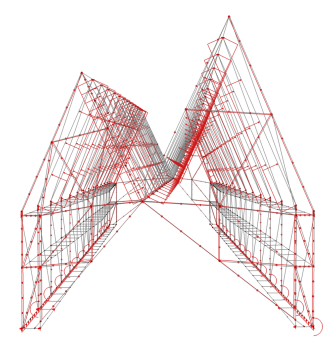

Wind (extra)

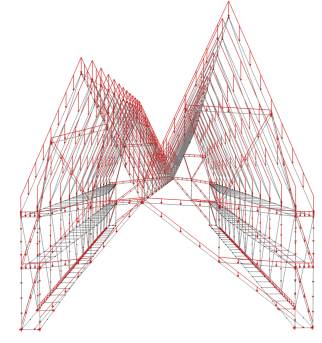

Permanent load

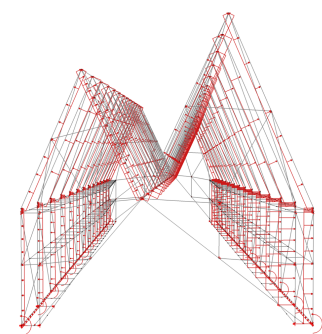

Wind $(\mathrm{N})$

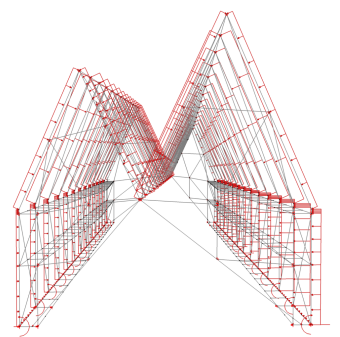

Wind (W)

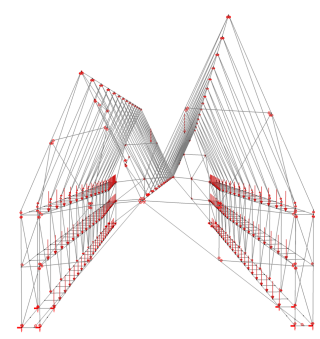

Lighting

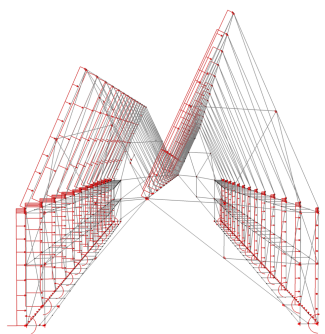

Wind $(\mathrm{E})$

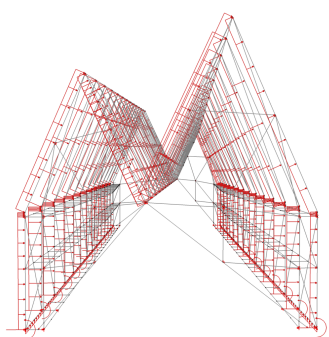

Wind (side)

Figure D.28: Load cases for the Market Hall. 